ARTICLE

https://doi.org/10.1038/s41467-019-11718-4

\title{
Tumor exosome-based nanoparticles are efficient drug carriers for chemotherapy
}

Tuying Yong $\mathbb{1}^{1,2,6}$, Xiaoqiong Zhang ${ }^{1,6}$, Nana Bie ${ }^{1,6}$, Hongbo Zhang ${ }^{3,6}$, Xuting Zhang $\mathbb{D}^{1}$, Fuying $\mathrm{Li}^{1}$, Abdul Hakeem', Jun Hu', Lu Gan (1) 1,2, Hélder A. Santos (1) ${ }^{4,5}$ \& Xiangliang Yang ${ }^{1,2}$

Developing biomimetic nanoparticles without loss of the integrity of proteins remains a major challenge in cancer chemotherapy. Here, we develop a biocompatible tumor-cell-exocytosed exosome-biomimetic porous silicon nanoparticles (PSiNPs) as drug carrier for targeted cancer chemotherapy. Exosome-sheathed doxorubicin-loaded PSiNPs (DOX@E-PSiNPs), generated by exocytosis of the endocytosed DOX-loaded PSiNPs from tumor cells, exhibit enhanced tumor accumulation, extravasation from blood vessels and penetration into deep tumor parenchyma following intravenous administration. In addition, DOX@E-PSiNPs, regardless of their origin, possess significant cellular uptake and cytotoxicity in both bulk cancer cells and cancer stem cells (CSCs). These properties endow DOX@E-PSiNPs with great in vivo enrichment in total tumor cells and side population cells with features of CSCs, resulting in anticancer activity and CSCs reduction in subcutaneous, orthotopic and metastatic tumor models. These results provide a proof-of-concept for the use of exosomebiomimetic nanoparticles exocytosed from tumor cells as a promising drug carrier for efficient cancer chemotherapy.

\footnotetext{
${ }^{1}$ National Engineering Research Center for Nanomedicine, College of Life Science and Technology, Huazhong University of Science and Technology, 430074 Wuhan, China. ${ }^{2}$ Hubei Key Laboratory of Bioinorganic Chemistry and Materia Medica, Huazhong University of Science and Technology, 430074 Wuhan, China. ${ }^{3}$ Pharmaceutical Sciences Laboratory and Turku Center for Biotechnology, Ảbo Akademi University, 20520 Turku, Finland. ${ }^{4}$ Drug Research Program, Division of Pharmaceutical Chemistry and Technology, University of Helsinki, FI-00014 Helsinki, Finland. ${ }^{5}$ Helsinki Institute of Life Science, University of Helsinki, FI-00014 Helsinki, Finland. ${ }^{6}$ These authors contributed equally: Tuying Yong, Xiaogiong Zhang, Nana Bie, Hongbo Zhang. Correspondence and requests for materials should be addressed to L.G. (email: lugan@mail.hust.edu.cn) or to H.A.S. (email: helder.santos@helsinki.fi) or to X.Y. (email: yangxl@mail.hust.edu.cn)
} 
$\mathrm{N}$ anoparticles-based drug delivery systems (NDDSs) have shown promising therapeutic efficacy in cancer due to the enhanced permeability and retention (EPR) effect ${ }^{1,2}$. To increase the capacity of targeting delivery of anticancer drugs to tumors, nanoparticles are usually functionalized with targeted antibodies, peptides or other biomolecules ${ }^{3,4}$. However, the presence of targeting ligands may sometimes have a negative influence on nanoparticle delivery owing to the enhanced immuneelimination ${ }^{5}$. Moreover, the targeting of these functionalized nanoparticles using targeting ligands is not possible and not precise for a wide range of cancers, because the receptors differ from versatile genetic or phenotypic heterogeneity of tumors ${ }^{6,7}$.

Biomimetic nanoparticles that combine the unique functionalities of natural biomaterials, such as cells or cell membranes, and engineering versatility of synthetic nanoparticles have recently increased considerable attention as effective drug delivery platforms ${ }^{8,9}$. Nanoparticles can be coated by various cell membranes from red blood cells (RBCs) ${ }^{10,11}$, cancer cells ${ }^{12,13}$, platelets $^{14}$, or white blood cells (WBCs) ${ }^{15}$, and have displayed good biocompatibility, prolonged circulation, as well as tumortargeting capacity. Exosomes are small extracellular vesicles secreted by mammalian cells ${ }^{16}$, and have lately been used as attractive nanocarriers owing to their stability in circulation, biocompatibility, low immunogenicity and low toxicity ${ }^{17-19}$. Furthermore, the exosomes display efficient cellular uptake and target-homing capabilities dependent on the proteins of their membrane ${ }^{17-19}$. Given that the surface protein composition of exosomes may be crucial to their function, preservation of exosome membrane integrity and stability is very important for their application in drug delivery ${ }^{20}$. Generally, exosomes-biomimetic nanoparticles are constructed by iterative physical extrusion or freeze/thaw cycles to fuse exosomes and nanoparticles ${ }^{21,22}$, which might affect the protein integrity on exosome membranes, thereby compromising the biofunctions of these biomimetic nanoparticles 23,24 . Therefore, it is highly desired to develop an efficient approach to construct exosome-biomimetic nanoparticles without interfering with the membrane integrity for cancer therapy.

Luminescent porous silicon nanoparticles (PSiNPs) have been widely used as drug carriers owing to their excellent drug loading capacity, high biocompatibility and biodegradability $15,25-30$. Here, we develop a biocompatible tumor cell-exocytosed exosome-sheathed PSiNPs (E-PSiNPs) as a drug carrier for targeted cancer chemotherapy. When tumor cells are incubated with doxorubicin-loaded PSiNPs (DOX@PSiNPs), they exocytose exosome-sheathed DOX-loaded PSiNPs (DOX@E-PSiNPs) (Fig. 1a). DOX@E-PSiNPs, regardless of the exosome origin, possess strong cross-reactivity of cellular uptake and cytotoxicity against bulk cancer cells and cancer stem cells (CSCs), which are responsible for tumorgenesis, tumor progression, recurrence, metastasis and drug resistance ${ }^{31,32}$. Moreover, DOX@E-PSiNPs exhibit enhanced tumor accumulation, extravasation from blood vessels and deep penetration into tumor parenchyma. These features of DOX@E-PSiNPs result in their greater in vivo enrichment in total tumor cells and side population cells with characteristics of $\mathrm{CSCs}^{33,34}$, thus generating remarkable anticancer and CSCs killing activity in subcutaneous, orthotopic and metastatic tumors (Fig. 1b). Our study provides a approach for cancer therapy by using exosome-biomimetic nanoparticles exocytosed from tumor cells as drug carriers to efficiently deliver anticancer drug.

\section{Results}

Autophagy-involved in the exocytosis of PSiNPs. Luminescent PSiNPs were prepared by electrochemical etching of silicon wafers, lift-off of PSi film, ultrasonication, centrifugation and finally activation of luminescence by heating in an aqueous solution. The hydrodynamic diameter of PSiNPs was ca. $150 \mathrm{~nm}$ measured by dynamic light scattering (DLS, Supplementary Fig. 1a). Scanning electron microscope (SEM) image showed a meso-porous nanostructure of the PSi film with the pore diameter of ca. $11 \mathrm{~nm}$ (Supplementary Fig. 1b). The BET surface area, pore volume and average pore diameter of PSiNPs were $211.8 \mathrm{~m}^{2} \mathrm{~g}^{-1}$, $0.2 \mathrm{~cm}^{2} \mathrm{~g}^{-1}$, and $13.5 \mathrm{~nm}$ as measured by nitrogen adsorption analysis (Supplementary Fig. 1c), respectively. The intrinsic photoluminescence of PSiNPs under $488 \mathrm{~nm}$ excitation appeared at wavelengths between 600 and $800 \mathrm{~nm}$ (Supplementary Fig. 1d).

Human hepatocarcinoma Bel7402 cells were treated with PSiNPs for $6 \mathrm{~h}$, followed by washing thoroughly with PBS and then incubating in fresh nanoparticle-free medium for different time intervals. Inductively coupled plasma-optical emission spectroscopy (ICP-OES) analysis showed that PSiNPs were exocytosed from Bel7402 cells in a time-dependent manner, and ca. $96 \%$ of PSiNPs were expelled out after culture in fresh medium for $18 \mathrm{~h}$ (Supplementary Fig. 2). Autophagy is a highly regulated process for intracellular homeostasis through clearance, degradation, or exocytosis of damaged cell components or foreign risks ${ }^{35}$. Thus, the exocytosis of PSiNPs may have high relevance to autophagy. To elucidate the role of autophagy in the exocytosis of PSiNPs, we first sought to determine whether PSiNPS-induced autophagy. Bel7402 cells were treated with PSiNPs for different time intervals and then the ratio of endogenous microtubuleassociated protein 1 light chain 3 (LC3)-II to LC3-I was assessed, since cytosolic LC3-I is conjugated to phosphatidylethanolamine to form membrane-associated LC3-II, which is recruited to autophagosomal membranes during autophagy, and therefore the conversion of LC3-I to LC3-II is considered to be an accurate indicator of autophagic activity ${ }^{36}$. PSiNPs treatment resulted in an increase of the ratio of LC3-II to LC3-I in a time-dependent manner (Fig. 2a), suggesting that PSiNPs treatment induces a cumulative increase in the formation of autophagosomes. Similar result was observed in murine hepatocarcinoma H22 cells (Supplementary Fig. 3), revealing that this phenomenal was not cell dependent. Bel7402 cells were also transfected with EGFPLC3 plasmid and then treated with PSiNPs for different time intervals. Consistently, treatment with PSiNPs led to significantly enhanced puncta formation of LC3-labeled vacuoles (Fig. 2b), confirming that PSiNPs-induced autophagosome formation. Moreover, we observed intracellular PSiNPs captured in the $\mathrm{LC}^{+}$autophagosomes (Fig. 2b). To determine whether autophagy was involved in the exocytosis of PSiNPs, we exposed Bel7402 cells with PSiNPs in the presence or absence of autophagy inhibitor 3-methyladenine (3-MA), or autophagy inducers rapamycin and carbamazepine (CBZ). 3-MA significantly inhibited the exocytosis of PSiNPs, while both rapamycin and CBZ significantly enhanced the exocytosis of PSiNPs (Fig. 2c), indicating that autophagy mediates the exocytosis of PSiNPs. Furthermore, the exocytosis of PSiNPs from Atg7 (a crucial autophagy gene)-deficient (Atg7 ${ }^{-/-}$) mouse embryonic fibroblasts (MEFs) was significantly lower than that from wild type MEFs (Fig. 2d), confirming that PSiNPs-induced autophagy regulates their exocytosis after internalization.

Exosomes sheathed with PSiNPs (E-PSiNPs). After Bel7402 cells were incubated with PSiNPs, we collected the exocytosed PSiNPS (E-PSiNPs) by centrifugation. Field transmission electron microscope (FTEM) energy spectrum analysis showed that silicon was detected in E-PSiNPs (Supplementary Fig. 4), endorsing that E-PSiNPs were actually the exocytosed PSiNPs. DLS analysis showed that the size of E-PSiNPs and PSiNPs was $260 \pm 15 \mathrm{~nm}$ 


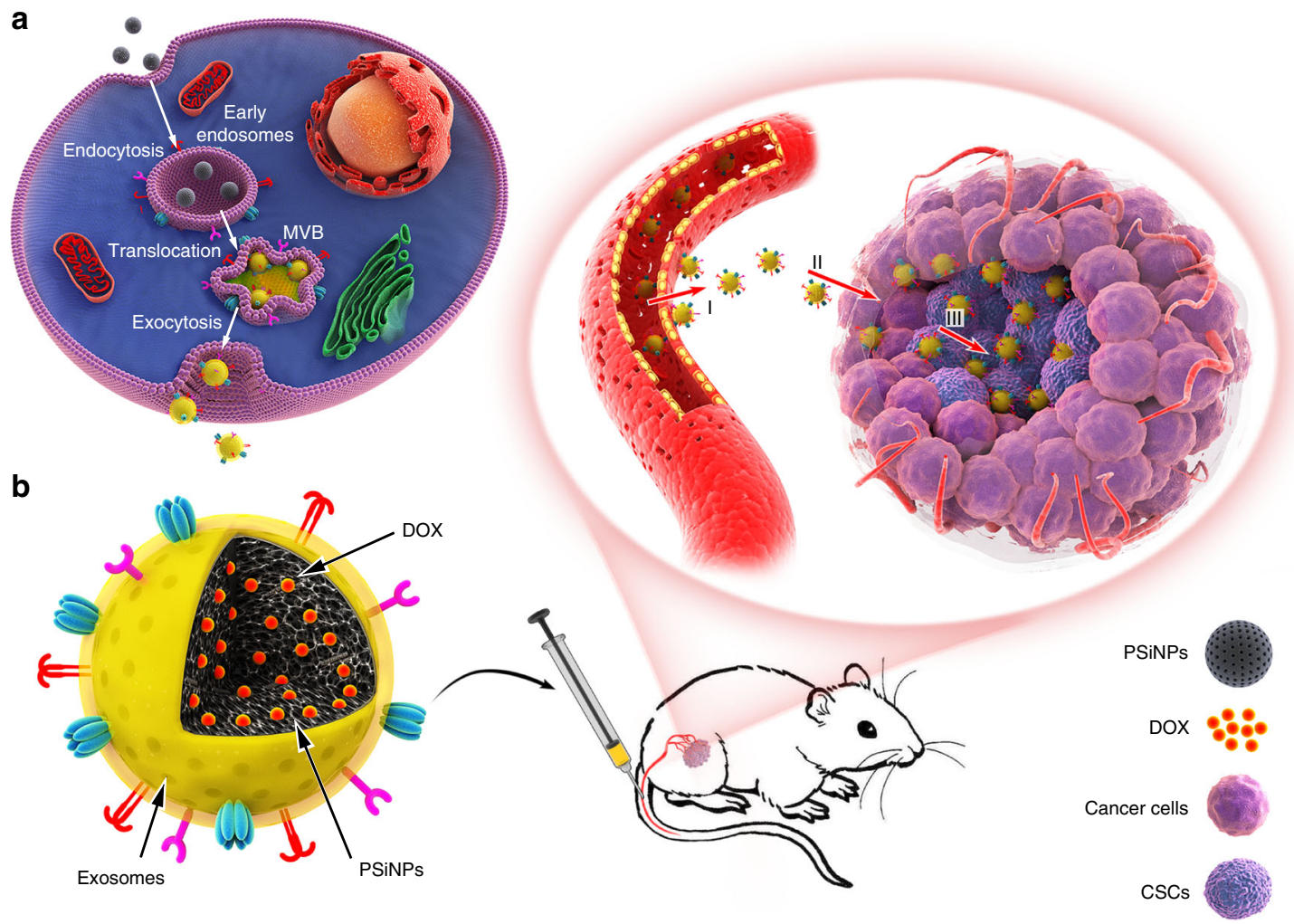

Fig. 1 Schematic illustration of E-PSiNPs as drug carriers for targeted cancer chemotherapy. a Schematic illustration of the preparation of DOX@E-PSiNPs. DOX@PSiNPs are endocytosed into cancer cells after incubation, then localized in multivesicular bodies (MVBs) and autophagosomes. After MVBs or amphisomes fuse with cell membrane, DOX@E-PSiNPs are exocytosed into extracellular space. b Schematics showing how DOX@E-PSiNPs effeciently target tumor cells after intravenous injection into tumor-bearing mice. (I) DOX@E-PSiNPs effeciently accumulate in tumor tissues; (II) DOX@E-PSiNPs penetrate deeply into tumor parenchyma; and (III) DOX@E-PSiNPs are efficently internalized into bulk cancer cells and CSCs to produce strong anticancer efficacy

and $150 \pm 11 \mathrm{~nm}$, and the corresponding PDI was $0.145 \pm 0.032$ and $0.208 \pm 0.028$, respectively (Fig. 3a). The zeta-potential of E-PSiNPs and PSiNPs was $-11.0 \pm 0.4 \mathrm{mV}$ and $-10.8 \pm 0.2 \mathrm{mV}$. TEM images revealed that PSiNPs and E-PSiNPs displayed irregular morphology, and ca. $20 \mathrm{~nm}$ thick membrane appeared on the surface of E-PSiNPs compared with PSiNPs (Fig. 3b). To further prove that PSiNPs were sheathed with membrane structure in E-PSiNPs, 3,3'-dioctadecyloxacarbocyanine perchlorate (DiO), a commonly used cell membrane fluorescent probe, was used to stain E-PSiNPs. Colocalization of green DiO fluorescence with intrinsic red PSiNPs fluorescence was observed in E-PSiNPs, but not in PSiNPs by confocal microscopy (Fig. 3c), confirming the presence of the membrane sheathed on PSiNPs in E-PSiNPs.

Exosomes, derived from fusion of intraluminal vesicles in MVBs with plasma membrane, serve as highly efficient export vehicles $^{17-19}$. When internalized into Bel7402 cells, PSiNPs were found to be colocalized with FITC-CD63-labeled MVBs (Supplementary Fig. 5), suggesting that PSiNPs are associated with MVBs before exocytosis. To explore whether E-PSiNPs were sheathed with exosomes, FITC-conjugated CD63 (a common biomarker for exosomes) antibody was used to label E-PSiNPs exocytosed from Bel7402 cells. As shown by immunofluorescent staining, CD63 was detected in E-PSiNPs, but not in PSiNPs (Fig. 3d). Western blot experiments further showed that similar to the whole cell lysates and the purified exosomes obtained by differential ultracentrifugation ${ }^{37,38}$, exosome biomarkers TSG101 and CD63 were also detected in E-PSiNPs (Fig. 3e), confirming the presence of exosomes in E-PSiNPs. In contrast to exosome biomarkers, calnexin, a protein located in endoplasmic reticulum $(\mathrm{ER})^{39}$, was only detected in whole cell lysates, but not in both
E-PSiNPs and the purified exosomes (Fig. 3e), revealing the high purity of the exosomes sheathed on PSiNPs in E-PSiNPs. Similar results were also observed in E-PSiNPs exocytosed from $\mathrm{H} 22$ cells (Supplementary Fig. 6), suggesting that E-PSiNPs can be generated from different cell lines. Moreover, dimethyl amiloride (DMA), an inhibitor of exosome release by disrupting calcium signaling 0 , was found to significantly inhibit the yield of E-PSiNPs, while ionomycin, a promoter of exosome release by increasing intracellular calcium concentration ${ }^{41}$, significantly augmented the yield of E-PSiNPs (Fig. 3f).

Overall, these results strongly confirm that the membrane that sheathed PSiNPs in E-PSiNPs is exosomes. The total protein amount of E-PSiNPs exocytosed from $10^{7}$ cancer cells was $60 \mu \mathrm{g}$, but only $1.8 \mu \mathrm{g}$ proteins were detected in the naturally secreted exosomes from equal numbers of cancer cells by differential ultracentrifugation, which was consistent with the other group's report $^{21}$. The fact that PSiNPs stimulated the production of exosomes by nearly 34 times shows that E-PSiNPs can be prepared with relatively high yield.

E-PSiNPs as a drug carrier. E-PSiNPs as a drug carrier were investigated using DOX as a model drug. DOX was loaded into PSiNPs and then incubated with Bel7402 cells. Exosome-sheathed DOX-loaded PSiNPs (DOX@E-PSiNPs) were also obtained by centrifugation in a similar fashion to E-PSiNPs. The colocalization of DOX, DiO-labeled membrane and PSiNPs by immunofluorescent staining showed the successful encapsulation of DOX into E-PSiNPs exocytosed from Bel7402 cells (Fig. 4a). DOX can also be encapsulated into E-PSiNPs exocytosed from $\mathrm{H} 22$ cells 
a
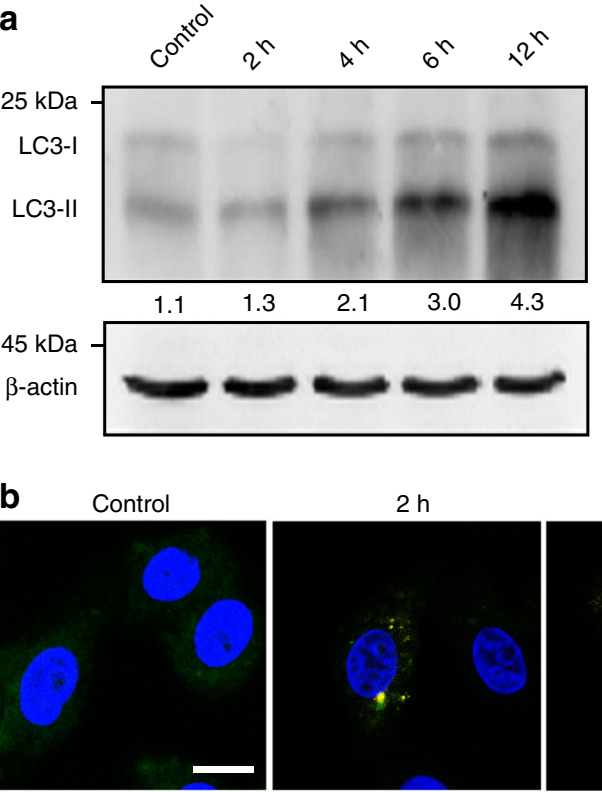

C

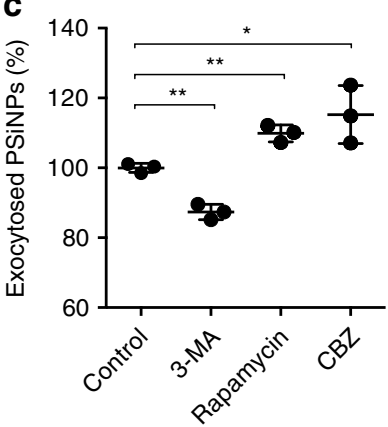

$4 \mathrm{~h}$

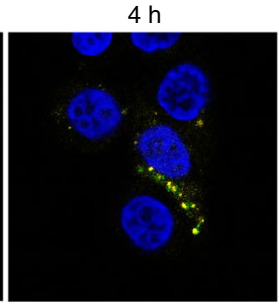

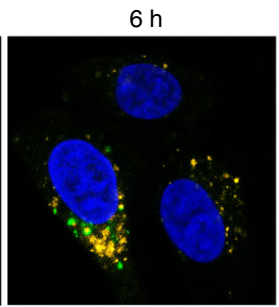

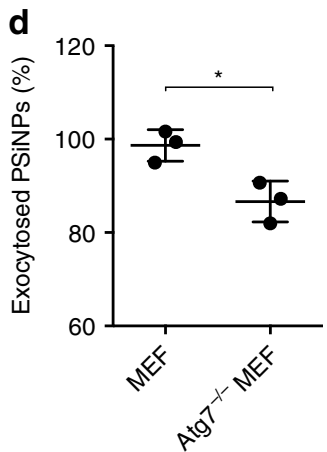

Fig. 2 Role of autophagy in the exocytosis of PSiNPs. a LC3-I and LC3-II expression in Bel7402 cells treated with $200 \mu \mathrm{g} \mathrm{mL}-1$ PSiNPs for different time intervals by western blot. The number underneath each group in the immunoblotting indicates the relative ratio of LC3-II to LC3-I of the corresponding group. b Confocal fluorescence microscopic images of EGFP-LC3-transfected Bel7402 cells after treatment with $200 \mu \mathrm{g} \mathrm{mL}-1$ PSiNPs for different time intervals. Scale bar: $20 \mu \mathrm{m}$. c Relative amount of the exocytosed PSiNPs in Bel7402 cells after treatment with $200 \mu \mathrm{g} \mathrm{mL}{ }^{-1}$ PSiNPs for $6 \mathrm{~h}$, followed by washing with PBS and then incubating in fresh medium with or without $5 \mathrm{mM}$ of 3-MA, $200 \mathrm{nM}$ of rapamycin or $30 \mu \mathrm{M}$ of $\mathrm{CBZ}$ for another $16 \mathrm{~h}$ by ICPOES. d Relative amount of the exocytosed PSiNPs in wild type and Atg7-/- MEF cells after treatment with $200 \mu \mathrm{mg} \mathrm{mL}^{-1} \mathrm{PSiNPs}$ for $6 \mathrm{~h}$, followed by washing with PBS and then incubating in fresh medium for $16 \mathrm{~h}$ by ICP-OES. Data were represented as mean \pm SD $\left(n=3\right.$ ). ${ }^{\star} P<0.05$, ${ }^{\star \star} P<0.01$ (one-way ANOVA with Fisher's LSD test for $\mathbf{c}$ and unpaired two-tailed Student's $t$ test for $\mathbf{d}$ ). Source data are provided as a Source Data file

using the same processing method (Supplementary Fig. 7). The drug loading degree of DOX@E-PSiNPs was $300 \mathrm{ng}$ DOX $\mu \mathrm{g}^{-1}$ protein (exosomes were quantified according to the protein content) and the drug loading efficiency was $0.8 \%$ determined by high performance liquid chromatography (HPLC). DOX loading did not significantly change the size of E-PSiNPs (Fig. 4b). Moreover, the size of DOX@E-PSiNPs remained almost constant even after incubating in PBS with or without $10 \%$ fetal bovine serum (FBS) for 6 days (Fig. 4c). Furthermore, storage at $-80^{\circ} \mathrm{C}$ for 1 month or lyophilization followed by resuspension in PBS 1 week later did not affect the size (Supplementary Fig. 8a, d) and zeta-potential (Supplementary Fig. 8b, e) of DOX@E-PSiNPs. Furthermore, relatively little degradation (Fig. 4d) and no significant morphology change (Supplementary Fig. 9) of DOX@EPSiNPs were detected after $72 \mathrm{~h}$ incubation in PBS. These results demonstrate that DOX@E-PSiNPs are relatively stable. DOX@EPSiNPs showed a sustained drug release profile as compared to DOX@PSiNPs (Fig. 4e), which can avoid the side effects caused by DOX burst release during blood circulation.

Efficient cellular uptake and cytotoxicity. To explore the biological function of DOX@E-PSiNPs, the interaction of DOX@EPSiNPs with CSCs with high drug resistance was first investigated. The H22 CSCs tumor spheroids were selected by the previously reported soft three-dimensional (3D) fibrin gel method $^{42,43}$. Intracellular DOX fluorescence increased in a dosedependent manner in H22 CSCs treated with free DOX, DOX@PSiNPs or DOX@E-PSiNPs exocytosed from H22 cells (Fig. 5a). However, DOX@E-PSiNPs displayed the highest intracellular accumulation, which was ca. 2.1 and 1.7 times more than free DOX and DOX@PSiNPs, respectively (Fig. 5a). DOX@E-PSiNPs after storage at $-80^{\circ} \mathrm{C}$ for 1 month or lyophilization followed by resuspension in PBS 1 week later still exhibited similarly strong cellular uptake by H22 CSCs (Supplementary Fig. 8c, f). Furthermore, the intracellular DOX retention in $\mathrm{H} 22$ CSCs was determined after treatment with free DOX, DOX@PSiNPs or DOX@E-PSiNPs exocytosed from H22 cells for $2 \mathrm{~h}$, followed by washing with PBS and then incubating in fresh medium for different time intervals. Treatment with DOX@EPSiNPs resulted in the enhanced DOX retention in H22 CSCs compared with free DOX or DOX@PSiNPs (Supplementary Fig. 10a). The enhanced DOX retention in DOX@E-PSiNPstreated H22 CSCs might be due to the decreased expression of multidrug-resistant protein P-glycoprotein (P-gp) (Supplementary Fig. 10b), a plasma membrane transporter whose expression was associated with cell membrane microenvironment ${ }^{44}$. DOX@E-PSiNPs-induced decrease in P-gp expression might be due to the strong interaction with cell membrane (Supplementary Fig. 11a, b), reducing the cell membrane fluidity (Supplementary Fig. 11c). Correspondingly, fewer H22 tumor spheroids were formed when $\mathrm{H} 22$ cells were pretreated with DOX@E-PSiNPs exocytosed from $\mathrm{H} 22$ cells for $4 \mathrm{~h}$ and then seeded in soft 3D fibrin gels ( $90 \mathrm{~Pa}, 400$ cells per well) for 5 days as compared to those pretreated with free DOX or DOX@PSiNPs (Fig. 5b). Moreover, colony sizes were reduced significantly in DOX@EPSiNPs-pretreated group (Fig. 5c). On the other hand, when $\mathrm{H} 22$ CSCs selected by soft 3D fibrin gels were treated with free DOX, DOX@PSiNPs or DOX@E-PSiNPs exocytosed from H22 cells for 24 h, DOX@E-PSiNPs also exhibited the strongest inhibition in colony number and size of tumor spheroids (Supplementary Fig. 12a, b). These results strongly suggest that DOX@E-PSiNPs display strong cellular uptake and intracellular retention with an excellent cytotoxicity against CSCs.

To evaluate whether DOX@E-PSiNPs possess cross-reactive cellular uptake and cytotoxicity, murine melanoma B16-F10 CSCs were treated with free DOX, DOX@PSiNPs or DOX@EPSiNPs exocytosed from H22 cells. Consistently, DOX@E- 

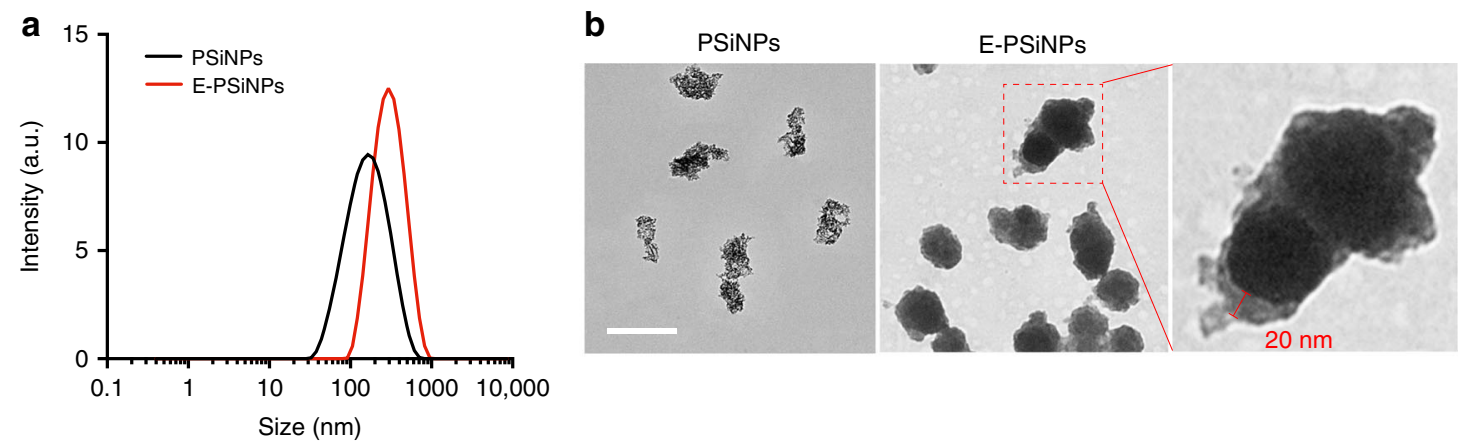

C
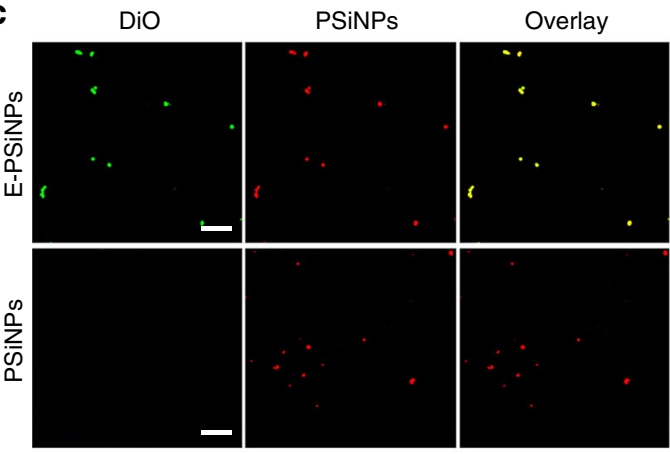

d

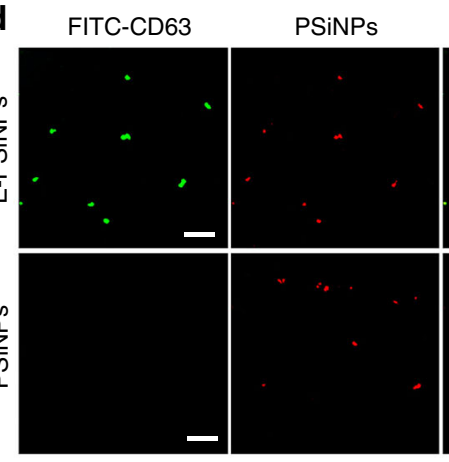

Overlay
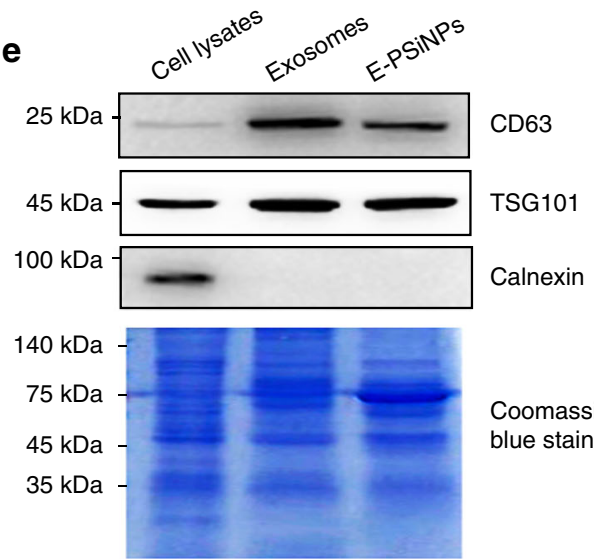

Coomassie

blue staining

$\mathbf{f}$

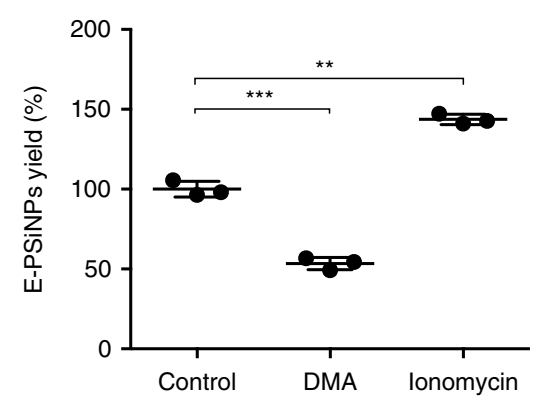

Fig. 3 Evaluation of exosomes sheathed on PSiNPs in E-PSiNPs. a Hydrodynamic diameter of PSiNPs and E-PSiNPs by DLS analysis. $\mathbf{b}$ TEM images of PSiNPs and E-PSiNPs. Scale bar: $200 \mathrm{~nm}$. c Colocalization of DiO (green) and PSiNPs (red) in E-PSiNPs by confocal microscopy. Scale bar: $20 \mu \mathrm{m}$. d Colocalization of CD63 (green) and PSiNPs (red) in E-PSiNPs by confocal microscopy. Scale bar: $20 \mu \mathrm{m}$. e Immunoblotting analysis of exosome markers (TSG101 and CD63) and ER marker (calnexin) expressed in E-PSiNPs exocytosed from Bel7402 cells. f Yield of E-PSiNPs when Bel7402 cells were pretreated with $200 \mu \mathrm{g} \mathrm{mL}-1$ PSiNPs for $6 \mathrm{~h}$ and then incubated in fresh medium containing $15 \mathrm{nM}$ DMA or $10 \mu \mathrm{M}$ ionomycin for $16 \mathrm{~h}$ by ICP-OES. Data were represented as mean \pm SD $(n=3)$. ${ }^{\star \star} P<0.01,{ }^{\star \star \star} P<0.001$ (one-way ANOVA with Fisher's LSD test). Source data are provided as a Source Data file

PSiNPs showed the highest internalization into B16-F10 CSCs (Fig. 5d) and had the corresponding strongest cytotoxicity against B16-F10 CSCs compared with free DOX or DOX@PSiNPs (Fig. 5e, f and Supplementary Fig. 13a, b). Similarly, DOX@EPSiNPs exocytosed from B16-F10 cells exhibited the strongest cellular uptake and cytotoxicity against H22 CSCs (Supplementary Fig. 14). These results suggest that DOX@E-PSiNPs have a strong cross-reactive cellular uptake and cytotoxicity against CSCs, irrespective of their origin. Furthermore, DOX@E-PSiNPs also demonstrated the highest intracellular internalization and cross-reactive cytotoxicity against bulk cancer cells, such as H22, Bel7402 and B16-F10 cells compared with free DOX or DOX@PSiNPs (Supplementary Fig. 15, 16). Intracellular trafficking analysis of DOX@E-PSiNPs revealed that exosomes and DOX were internalized into cancer cells together and then colocalized with lysosomes, followed by DOX translocation to nuclei over time (Supplementary Fig. 17). Considering that more DOX was released from DOX@E-PSiNPs under lysosomal acidic $\mathrm{pH}$ (Supplementary Fig. 18), DOX@E-PSiNPs released DOX in lysosomes to enter nuclei to exert the cytotoxicity. CD54 (ICAM1), a member of the immunoglobulin supergene family, was found to be involved in the cross-reactive cellular uptake of DOX@E-PSiNPs by cancer cells, as evidenced by the fact that DOX@E-PSiNPs exocytosed from B16-F10 and H22 cells expressed CD54 (Supplementary Fig. 19a), and pretreatment with CD54 antibody decreased the cellular uptake of DOX@EPSiNPs exocytosed from H22 cells by $\mathrm{H} 22$ and B16-F10 cells (Supplementary Fig. 19b). Despite the strong cellular uptake by tumor cells, DOX@E-PSiNPs exocytosed from H22 cells exhibited less internalization into human umbilical vein endothelial cells 
a
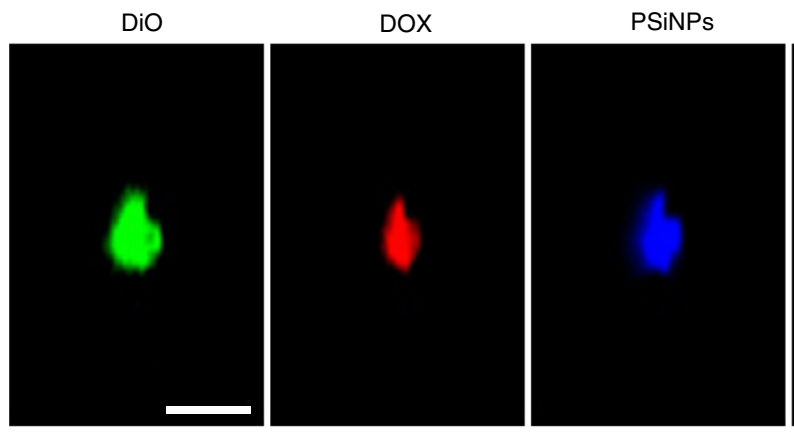

C

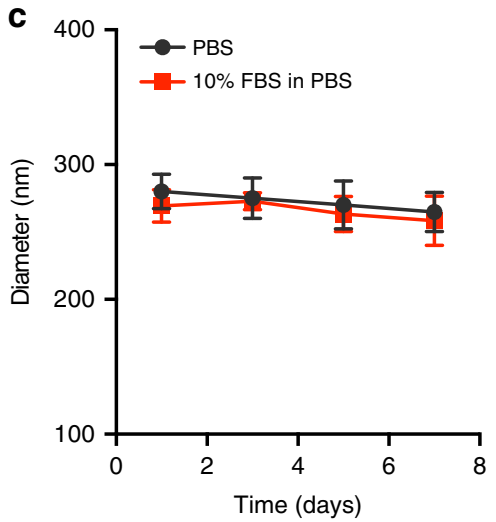

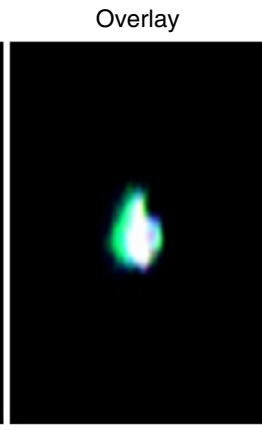

b
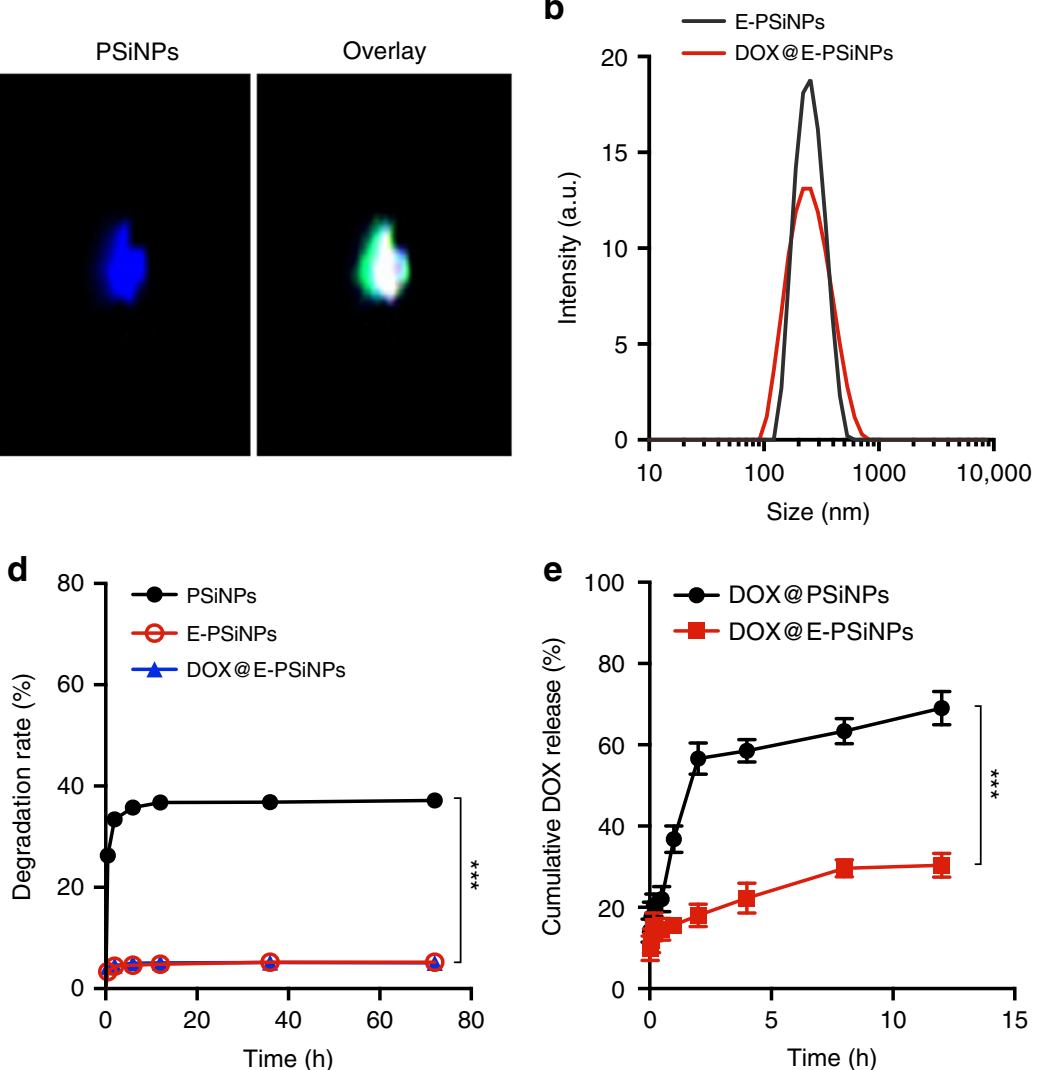

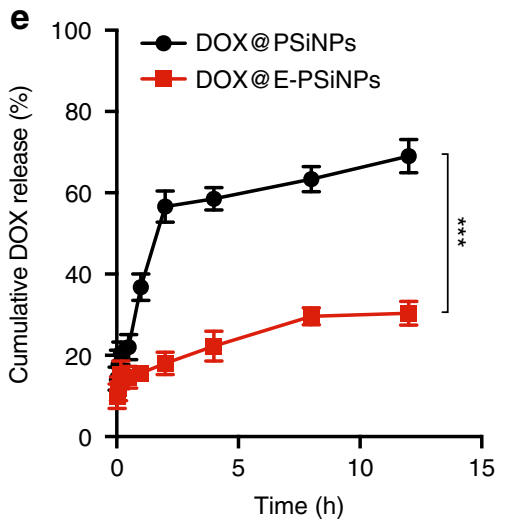

Fig. 4 Characterization of DOX@E-PSiNPs. a Colocalization of DiO, DOX, and PSiNPs in DOX@E-PSiNPs exocytosed from Bel7402 cells by confocal microscopy. Scale bar: $1 \mu \mathrm{m}$. b Hydrodynamic diameter of E-PSiNPs and DOX@E-PSiNPs by DLS. c Hydrodynamic diameter of E-PSiNPs incubating in PBS with or without $10 \%$ FBS for different time intervals. d Degradation behavior of PSiNPs, E-PSiNPs and DOX@E-PSiNPs in PBS at $37^{\circ} \mathrm{C}$. e In vitro DOX release profiles of DOX@PSiNPs and DOX@E-PSiNPs in PBS at pH 7.4 by dialysis bag. Data were presented as mean \pm SD $(n=3)$. ${ }^{\star \star \star} P<0.001$ (one-way ANOVA with Bonferroni's multiple comparisons test for $\mathbf{d}$ and unpaired two-tailed Student's $t$ test for $\mathbf{e}$ ). Source data are provided as a Source Data file

(HUVECs). In addition, less DOX@E-PSiNPs exocytosed from HUVECs cells were internalized into H22 cells compared with DOX@E-PSiNPs exocytosed from H22 cells (Supplementary Fig. 20), suggesting the tumor cell targeting capacity of tumor exosome-coated PSiNPs.

Enhanced tumor accumulation and penetration. Besides efficient cellular uptake and accompanied strong cytotoxicity against bulk cancer cells and CSCs, an ideal anticancer drug delivery system following systemic administration should be characterized by enhanced tumor accumulation and penetration to reach bulk cancer cells and CSCs. Therefore, the in vivo biodistribution of DOX@E-PSiNPs was investigated. Mice bearing H22 hepatocarcinoma tumors were intravenously injected with free DOX, DOX@PSiNPs or DOX@E-PSiNPs at $0.5 \mathrm{mg} \mathrm{kg}^{-1}$ DOX dosage, or high dosage of free DOX at $4 \mathrm{mg} \mathrm{kg}^{-1}$. At $24 \mathrm{~h}$ after injection, the tumors and major normal organs (heart, liver, spleen, lung and kidney) were collected for DOX content measurement. Although DOX@E-PSiNPs were accumulated in liver at relatively high level (Fig. 6a), especially Kupffer cells in liver (Supplementary Fig. 21), less DOX was accumulated in normal organs (heart, liver, lung and kidney) of DOX@E-PSiNPs-treated mice than that of high dosage of DOX-treated mice (Fig. 6a). However, DOX@EPSiNPs exhibited strong tumor tropism and accumulation, ca. 2.5 and 2.3 times relative to free DOX and DOX@PSiNPs, respectively, comparable to high dosage of free DOX (Fig. 6a). Pretreatment with CD54 antibody decreased the tumor accumulation of DOX@E-PSiNPs, suggesting that similar to the cross-reactive cellular uptake by cancer cells, CD54 was also involved in the enhanced tumor accumulation of DOX@E-PSiNPs (Supplementary Fig. 22). The strong tumor-targeting ability of DOX@EPSiNPs was further confirmed in B16-F10 lung metastatic model (Supplementary Fig. 23).

Furthermore, we addressed the tumor penetration capacity of DOX@E-PSiNPs. First, tumor spheroids as in vivo-mimetic tumors were treated with free DOX, DOX@PSiNPs or DOX@EPSiNPs for $24 \mathrm{~h}$, and then the tumor spheroids were optically sectioned using confocal microscopy. The projection images of DOX fluorescence in tumor spheroids was reconstructed by using Amira software. DOX fluorescence intensity in both $X$ - and $Y$ axis shadows was distinctly stronger in DOX@E-PSiNPs-treated group than that in free DOX- or DOX@PSiNPs-treated group at the same depth (Supplementary Fig. 24), suggesting the deep tumor penetration ability of DOX@E-PSiNPs. Furthermore, the deep tumor penetration capability of DOX@E-PSiNPs was investigated in $\mathrm{H} 22$ tumor-bearing mice by intravenous injection of free DOX, DOX@PSiNPs or DOX@E-PSiNPs. Confocal fluorescence microscopic images clearly showed that DOX@EPSiNPs were distributed widely in whole tumor section at $24 \mathrm{~h}$ after injection (Fig. 6b). In contrast, DOX@PSiNPs and free DOX accumulated more around the blood vessels as indicated by stronger co-localization with FITC-CD31-labeled endothelial cells (Fig. 6b). The distance-dependent DOX fluorescence intensity also confirmed that only fluorescence signal of DOX delivered with DOX@E-PSiNPs was detectable at ca. $400 \mu \mathrm{m}$ far away from the blood vessels, while free DOX or DOX delivered with DOX@PSiNPs was found at $<120 \mu \mathrm{m}$ away from the blood vessels (Fig. 6c). Overall, these results show that DOX@E-PSiNPs are 

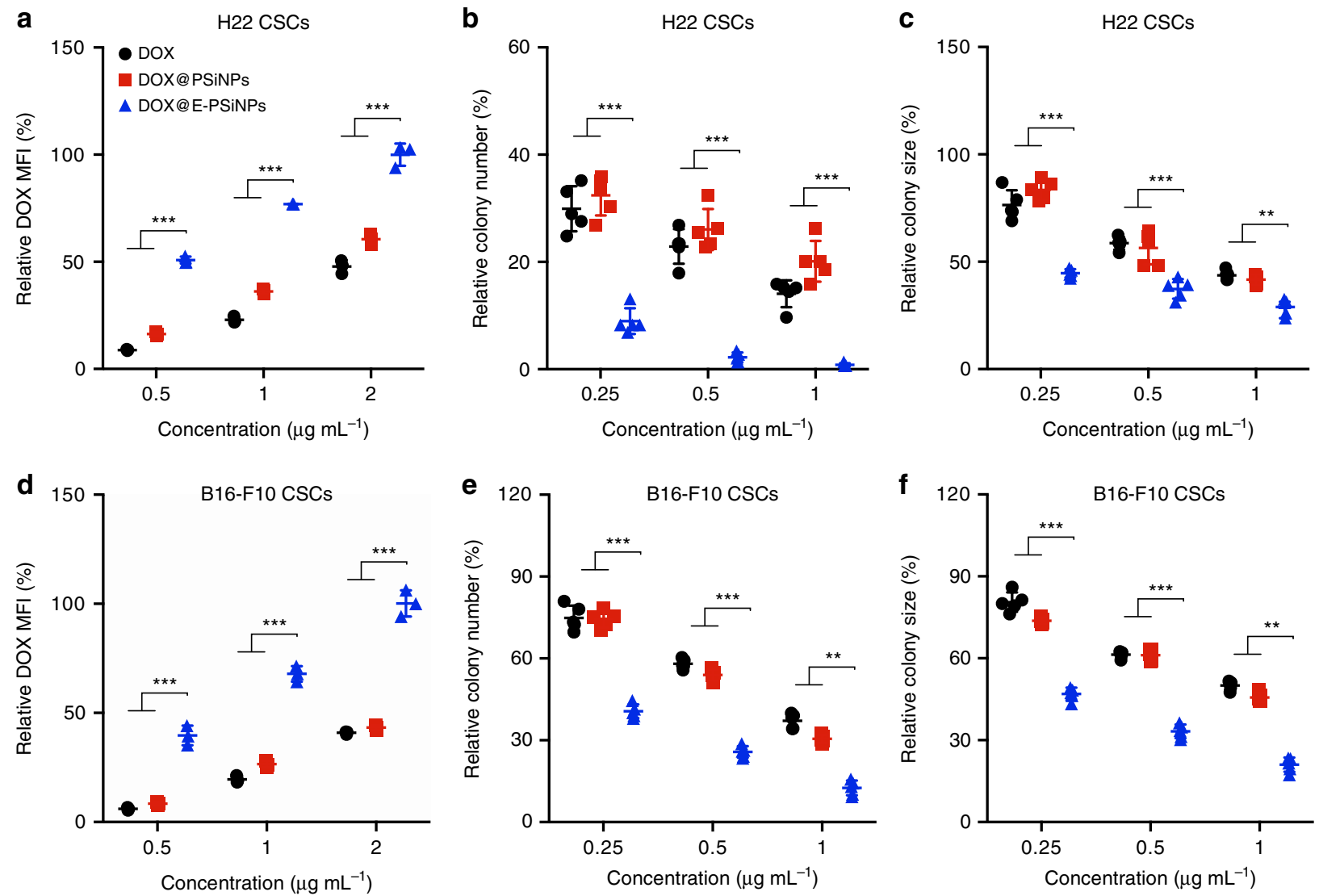

Fig. 5 Cellular uptake and cytotoxicity of DOX@E-PSiNPs against CSCs. a, d Relative DOX mean fluorescence intensity (MFI) when H22 CSCs (a) and B16F10 CSCs (d) selected in soft 3D fibrin gels were treated with free DOX, DOX@PSiNPs or DOX@E-PSiNPs exocytosed from H22 cells at different DOX concentrations for $2 \mathrm{~h}$ by flow cytometry. Data were represented as mean $\pm \mathrm{SD}(n=3)$. b, e Relative colony number of tumor spheroids when $\mathrm{H} 22(\mathbf{b})$ and B16-F10 cells (e) were pretreated with free DOX, DOX@PSiNPs or DOX@E-PSiNPs exocytosed from H22 cells at different DOX concentrations for $4 \mathrm{~h}$ and then seeded in soft 3D fibrin gels for 5 days. c, f Relative colony size of tumor spheroids when $\mathrm{H} 22$ (c) and B16-F10 cells (f) were pretreated with free DOX, DOX@PSiNPs or DOX@E-PSiNPs exocytosed from H22 cells at different DOX concentrations for $4 \mathrm{~h}$ and then seeded in soft 3D fibrin gels for 5 days. Data were represented as mean \pm SD $(n=5) .{ }^{\star \star} P<0.01,{ }^{\star \star \star} P<0.001$ (two-way ANOVA with Bonferroni's multiple comparisons test). Source data are provided as a Source Data file

easy to extravasate from the blood vessels and penetrate into deep tumor parenchyma. The strong intercellular delivery capacity of DOX@E-PSiNPs might be responsible for their enhanced tumor penetration $^{26}$ (Supplementary Fig. 25), which is regulated by CD54 expressed on exosomes of DOX@E-PSiNPs (Supplementary Fig. 26).

Enhanced in vivo enrichment in side population cells. Given that DOX@E-PSiNPs demonstrate enhanced tumor accumulation and penetration, as well as efficient cellular uptake by bulk cancer cells and CSCs, their in vivo enrichment in total tumor cells and CSCs might be improved. Therefore, we determined the in vivo DOX accumulation in total tumor cells at $24 \mathrm{~h}$ after GFPexpressing $\mathrm{H} 22$ tumor-bearing mice were intravenously injected with free DOX, DOX@PSiNPs or DOX@E-PSiNPs at DOX dosage of $0.5 \mathrm{mg} \mathrm{kg}^{-1}$, or high dosage of free DOX at $4 \mathrm{mg} \mathrm{kg}^{-1}$. The tumor tissues were digested into single cells and the intracellular DOX fluorescence in total GFP-positive tumor cells was measured by flow cytometry (Fig. 6d). DOX content in the total GFP-positive tumor cells of mice administrated with DOX@EPSiNPs was about 3.2 times of both free DOX- and DOX@PSiNPs-treated groups, respectively, even significantly higher than that of free DOX-treated group at high dosage. Subsequently, we isolated the side population cells from GFP-positive H22 tumors by flow cytometry and determined the intracellular DOX fluorescence (Fig. 6e). Similarly, higher DOX fluorescence intensity was detected in side population cells of DOX@EPSiNPs-treated mice compared with that of free DOX-, DOX@PSiNPs- or high dosage of free DOX-treated group. Collectively, these results reveal that DOX@E-PSiNPs exhibit augmented in vivo enrichment in the total tumor cells and side population cells after intravenous injection, which further increases their in vivo anticancer and CSCs killing activity.

Excellent anticancer and CSCs killing activity. The in vivo anticancer activity of DOX@E-PSiNPs was determined in BALB/c mice bearing subcutaneous H22 tumors. Free DOX, DOX@PSiNPs, DOX@E-PSiNPs exocytosed from H22 cells at DOX dosage of $0.5 \mathrm{mg} \mathrm{kg}^{-1}$, or free DOX at high dosage of $4 \mathrm{mg} \mathrm{kg}^{-1}$ were intravenously administrated into $\mathrm{H} 22$ tumor-bearing mice once every 3 days for 17 days. The tumors grew very fast, and free DOX and DOX@PSiNPs at $0.5 \mathrm{mg} \mathrm{kg}^{-1}$ dosage did not significantly inhibit the tumor growth compared with PBS and EPSiNPs (Fig. 7a, b and Supplementary Fig. 27). In contrast, DOX@E-PSiNPs at DOX dosage of $0.5 \mathrm{mg} \mathrm{kg}^{-1}$ showed a significant anticancer activity, with 91 and $87 \%$ reduction in tumor volume and tumor weight compared to the PBS group, respectively, and even stronger than free DOX at high dosage (Fig. 7a, b and Supplementary Fig. 27). The excellent anticancer activity of DOX@E-PSiNPs was further confirmed by increased 

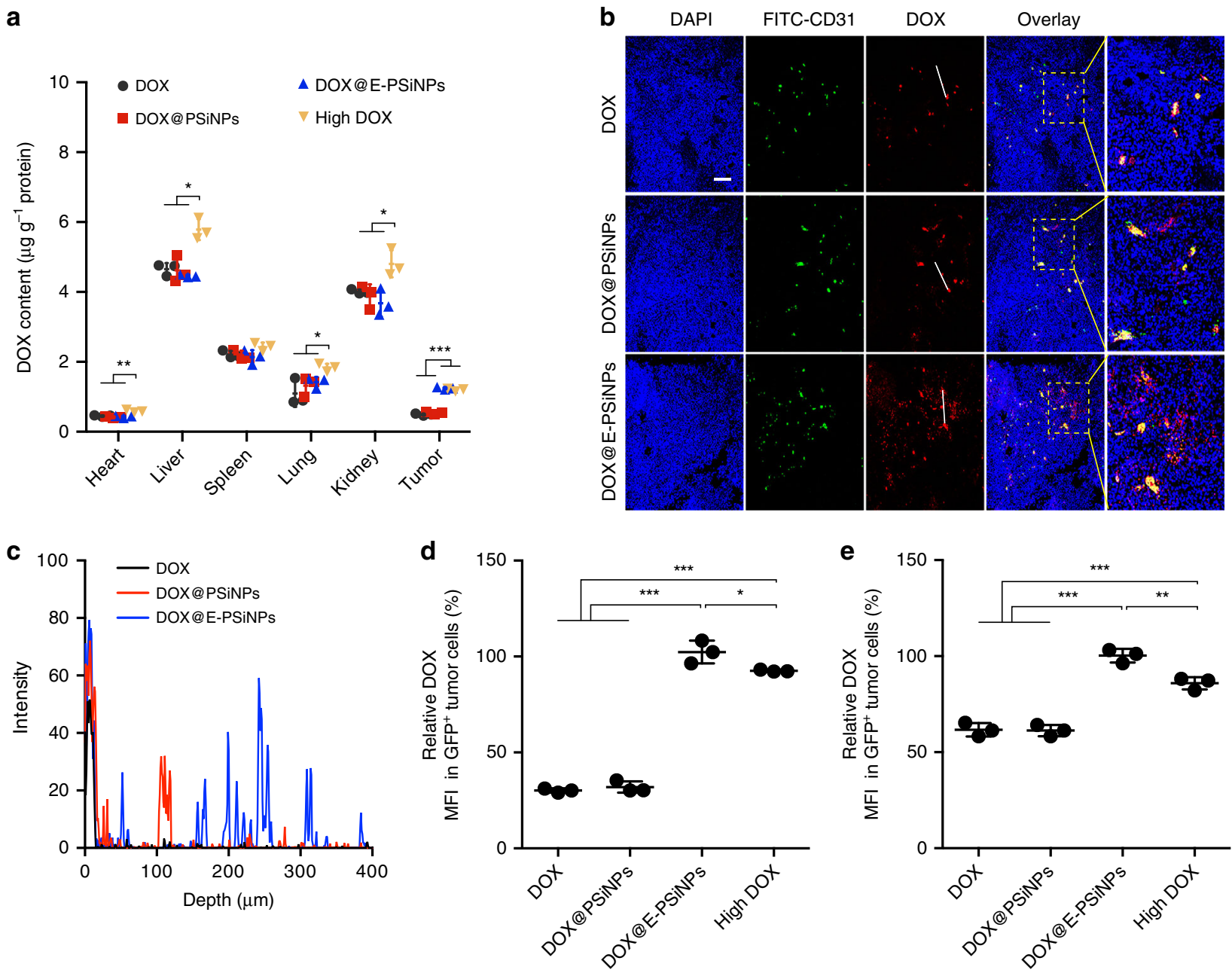

Fig. 6 Accumulation and penetration of DOX@E-PSiNPs into tumor parenchyma. a DOX content in tumor tissues and major organs of H22 tumor-bearing mice at 24 h after intravenous injection of DOX, DOX@PSiNPs or DOX@E-PSiNPs at DOX dosage of $0.5 \mathrm{mg} \mathrm{kg}^{-1}$, or high dosage of DOX at $4 \mathrm{mg} \mathrm{kg}^{-1}$. b Colocalization of DOX and CD31-labeled tumor vessels in tumor sections of $\mathrm{H} 22$ tumor-bearing mice at $24 \mathrm{~h}$ after intravenous injection of DOX, DOX@PSiNPs or DOX@E-PSiNPs at DOX dosage of $0.5 \mathrm{mg} \mathrm{kg}^{-1}$. Scale bar: $200 \mu \mathrm{m}$. White lines represent the distance between DOX in blood vessels and DOX in tumor parenchyma. c DOX distribution profile from the blood vessels to tumor tissues on the specified white lines as indicated in $\mathbf{b}$. $\mathbf{d}$, $\mathbf{e}$ Relative DOX fluorescence intensity in GFP-positive tumor cells (d) and side population cells (e) of tumor tissues at $24 \mathrm{~h}$ after GFP-expressing H22 tumor-bearing mice were intravenously injected with DOX, DOX@PSiNPs or DOX@E-PSiNPs at DOX dosage of $0.5 \mathrm{mg} \mathrm{kg}^{-1}$, or high dosage of DOX at $4 \mathrm{mg} \mathrm{kg}{ }^{-1}$. Data were represented as mean $\pm \mathrm{SD}(n=3) .{ }^{\star} P<0.05,{ }^{\star \star} P<0.01,{ }^{\star \star \star} P<0.001$ (two-way ANOVA with Bonferroni's multiple comparisons test for a and oneway ANOVA with Bonferroni's multiple comparisons test for $\mathbf{d}, \mathbf{e})$. Source data are provided as a Source Data file

TUNEL-positive apoptotic tumor cells in excised tumor tissues (Supplementary Fig. 28). Moreover, prolonged survival time was observed in $\mathrm{H} 22$ tumor-bearing mice treated with DOX@EPSiNPs (122 days), compared with PBS- (82 days), E-PSiNPs(85 days), free DOX- (84 days) or DOX@PSiNPs-treated group (87 days) at $0.5 \mathrm{mg} / \mathrm{kg}$ DOX dosage, or free DOX at $4 \mathrm{mg} / \mathrm{kg}$ dosage (109 days) (Fig. 7c). Importantly, DOX@E-PSiNPs did not show systemic toxicity to $\mathrm{H} 22$ tumor-bearing mice, as evidenced by body weight (Supplementary Fig. 29), hematoxylin-eosin $(\mathrm{H} \& \mathrm{E})$ staining of major organs (Supplementary Fig. 30) and serological analysis (Supplementary Fig. 31). However, free DOX at high dosage of $4 \mathrm{mg} \mathrm{kg}^{-1}$ induced cardiotoxicity (Supplementary Fig. 30, 31).

To further assess whether DOX@E-PSiNPs could efficiently kill CSCs, the tumor tissues after treatment were digested into single cells, and the number of CD133-positive cells (a CSC marker of liver cancer ${ }^{45}$ ) was measured. As a result, CD133-positive cells were significantly inhibited in DOX@E-PSiNPs-treated group, compared with free DOX-, DOX@PSiNPs-, or high dosage of free
DOX-treated group (Fig. 7d). Consistently, the number of side population cells in tumor tissues of DOX@E-PSiNPs-treated GFP-expressing H22 tumor-bearing mice was the fewest compared with other groups (Fig. 7e). Furthermore, 800 single tumor cells were seeded in soft $3 \mathrm{D}$ fibrin gels $(90 \mathrm{~Pa})$ for 5 days, which was developed to select $\mathrm{CSCs}^{42,43}$. The fewest colony number and smallest colony size were formed in DOX@EPSiNPs-treated group (Fig. 7f, g). The excellent CSCs killing activity of DOX@E-PSiNPs was further confirmed by subcutaneously transplanting the same amounts of tumor cells from tumor tissues after treatment into BALB/c mice (Fig. $7 \mathrm{~h}$ ). $100 \%$ of mice $(6 / 6$ mice) generated tumors at 6 days after secondary transplantation of tumor cells of PBS-, E-PSiNPs-, free DOX- or DOX@PSiNPs-treated group. However, 83\% (5/6 mice) and 33\% (2/6 mice) of mice generated tumors at 40 days after secondary transplantation of tumor cells of high dosage of DOX- and DOX@E-PSiNPs-treated groups, respectively. Taken together, these results strongly reveal that DOX@E-PSiNPs have excellent anticancer and CSCs killing activity. To further improve the 

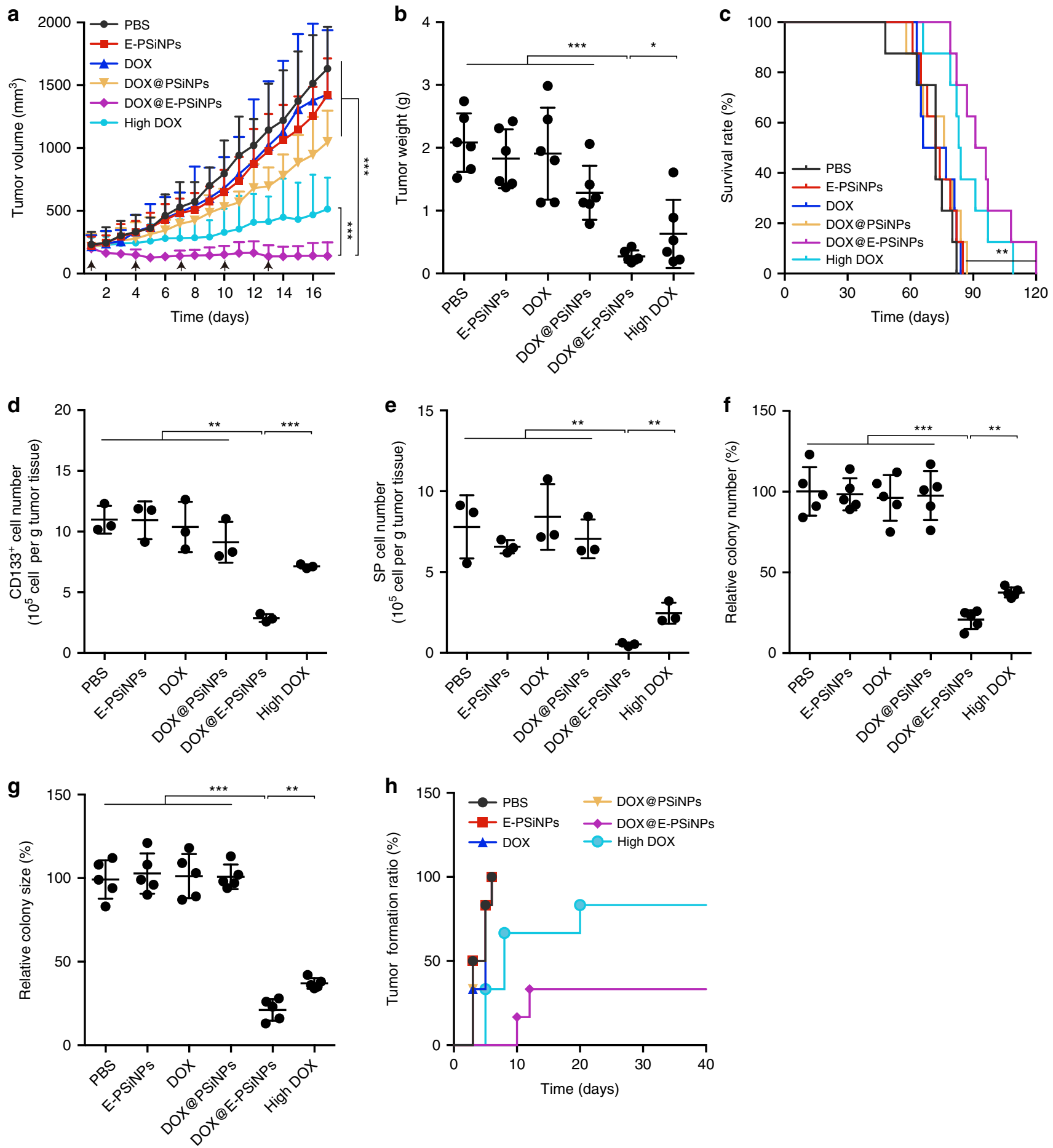

Fig. 7 Anticancer activity of DOX@E-PSiNPs in H22 tumor-bearing mice. a Tumor growth curves of H22 tumor-bearing mice after intravenous injection of PBS, E-PSiNPs, free DOX, DOX@PSiNPs, DOX@E-PSiNPs exocytosed from H22 cells at DOX dosage of $0.5 \mathrm{mg} \mathrm{kg}^{-1}$, or free DOX at high dosage of $4 \mathrm{mg} \mathrm{kg}{ }^{-1}$. The arrows indicate the drug injection time. Data were represented as mean \pm SD $(n=14)$. $\mathbf{b}$ Weight of tumor tissues at the end of tumor growth inhibition experiments. Data were represented as mean $\pm \mathrm{SD}(n=6)$. c Kaplan-Meier survival plot of $\mathrm{H} 22$ tumor-bearing mice after intravenous administration of different formulations $(n=8)$. $\mathbf{d}$ Number of CD133-postive cells in tumor tissues at the end of tumor growth inhibition experiments. e Number of side population cells in GFP-positive tumor cells of GFP-expressing H22 tumor-bearing mice at the end of tumor growth inhibition experiments as above. Data were represented as mean \pm SD $(n=3)$. $\mathbf{f}, \mathbf{g}$ Relative colony number $(\mathbf{f})$ and size $(\mathbf{g})$ of tumor spheroids when tumor cells digested from tumor tissues of H22 tumor-bearing mice at the end of tumor growth inhibition experiments were seeded in soft 3D fibrin gels for 5 days. Data were represented as mean \pm SD $(n=5)$. $\mathbf{h}$ Tumor formation ratio in $\mathrm{BALB} / \mathrm{c}$ mice after subcutaneous injection of tumor cells ( $10^{6}$ cells per mouse) from tumor tissues of $\mathrm{H} 22$ tumor-bearing mice after treatment as above. ${ }^{\star} P<0.05$, ${ }^{\star \star} P<0.01,{ }^{\star \star \star} P<0.001$ (one-way ANOVA with Bonferroni's multiple comparisons test for $\mathbf{a}, \mathbf{b}$, and $\mathbf{d}-\mathbf{g}$ and log-rank test for $\mathbf{c}$ ). Source data are provided as a Source Data file 
anticancer and CSCs killing efficacy, more DOX@E-PSiNPs at DOX dosage of $0.8 \mathrm{mg} \mathrm{kg}^{-1}$, or the combination of DOX@EPSiNPs at DOX dosage of $0.5 \mathrm{mg} \mathrm{kg}^{-1}$ and all-trans-retinoic acid (ATRA), a powerful differentiating agent of CSCs, were intravenously injected into $\mathrm{H} 22$ tumor-bearing mice. As expected, increasing the used dosage of DOX@E-PSiNPs, or combination of DOX@E-PSiNPs and ATRA resulted in a significant tumor inhibition, with 3 or 2 tumor ablation in 6 mice, respectively (Supplementary Fig. 32a-g). Correspondingly, fewer side population cells in tumor tissues (Supplementary Fig. 32h), fewer colony number (Supplementary Fig. 32i) and smaller colony size (Supplementary Fig. 32j) after seeding the tumor cells in 3D fibrin gels were observed in these groups compared with only DOX@E-PSiNPs treatment group at DOX dosage of $0.5 \mathrm{mg} \mathrm{kg}^{-1}$, suggesting that CSCs might be responsible for the drug resistance. Meanwhile, combination treatment of DOX@E-PSiNPs and ATRA, or increasing the used dosage of DOX@E-PSiNPs was found to be safe, as evidenced by routine blood test (Supplementary Fig. 33a-d), serological analysis (Supplementary Fig. 33e-j) and body weight (Supplementary Fig. 33k).

To further investigate the cross-reactive anticancer and CSCs killing efficacy of DOX@E-PSiNPs, mice bearing orthotopic
4T1 breast tumors were intravenously administrated with free DOX, DOX@PSiNPs, DOX@E-PSiNPs exocytosed from H22 cells at DOX dosage of $0.5 \mathrm{mg} \mathrm{kg}^{-1}$ or free DOX at high dosage of $4 \mathrm{mg} \mathrm{kg}^{-1}$ once every 3 days for 15 days. DOX@E-PSiNPs at DOX dosage of $0.5 \mathrm{mg} \mathrm{kg}^{-1}$ exhibited a significant anticancer activity, with $68 \%$ and $65 \%$ reduction in tumor volume and tumor weight compared to the PBS group, respectively (Fig. 8a, b). Mice treated with DOX@E-PSiNPs had 11, 10, and 4 days longer survival time as compared to free DOX and DOX@PSiNPs at DOX dosage of $0.5 \mathrm{mg} \mathrm{kg}^{-1}$, and free DOX at $4 \mathrm{mg} \mathrm{kg}^{-1}$ dosage (Fig. 8c). Furthermore, tumor cells digested from breast tumors after treatment were seeded in soft $3 \mathrm{D}$ fibrin gels $(90 \mathrm{~Pa})$. The fewest colony number and smallest colony size of the formed tumor spheroids were detected in DOX@E-PSiNPs-treated group (Fig. 8d, e). These results demonstrate the excellent cross-reactive anticancer and CSCs killing efficacy of DOX@E-PSiNPs. DOX@E-PSiNPs did not cause toxicity to $4 \mathrm{~T} 1$ tumor-bearing mice, as evidenced by routine blood test (Supplementary Fig. 34), serological analysis (Supplementary Fig. 35) and H\&E staining of major organs (Supplementary Fig. 36), although free DOX at $4 \mathrm{mg} \mathrm{kg}^{-1}$ dosage caused bone marrow and heart toxicity.
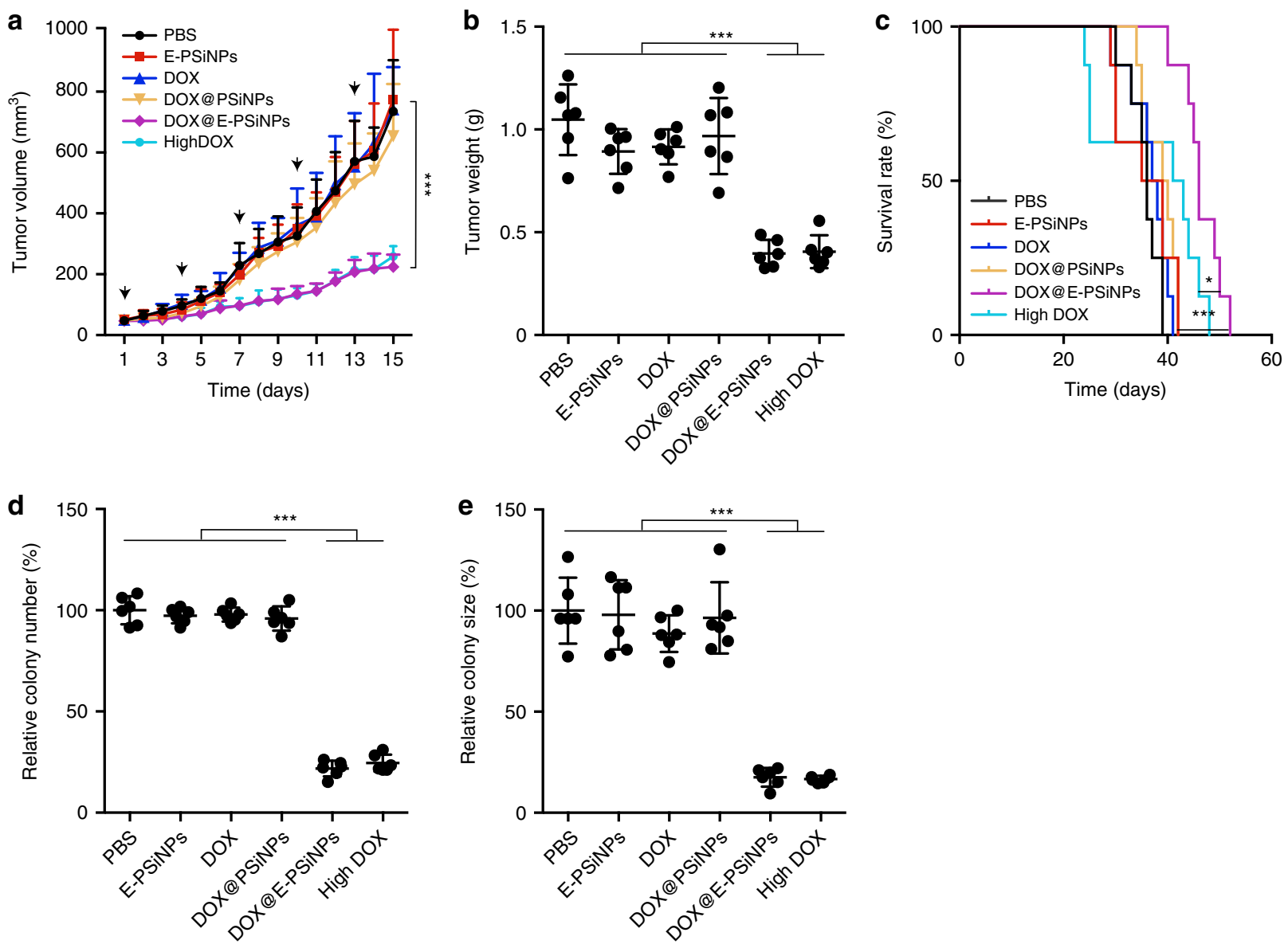

Fig. 8 Anticancer activity of DOX@E-PSiNPs in orthotopic 4T1 tumor-bearing mice. a Tumor growth curves of orthotopic 4T1 tumor-bearing mice after intravenous injection of PBS, E-PSiNPs, free DOX, DOX@PSiNPs, DOX@E-PSiNPs exocytosed from H22 cells at DOX dosage of 0.5 mg kg-1, or free DOX at high dosage of $4 \mathrm{mg} \mathrm{kg}^{-1}$. The arrows indicate the drug injection time. Data were represented as mean $\pm \mathrm{SD}(n=14)$. $\mathbf{b}$ Weight of tumor tissues at the end of tumor growth inhibition experiments. Data were represented as mean \pm SD $(n=6)$. c Kaplan-Meier survival plot of $4 \mathrm{~T} 1$ tumor-bearing mice after intravenous administration of different formulations $(n=8)$. $\mathbf{d}$, e Relative colony number $(\mathbf{d})$ and size $(\mathbf{e})$ of tumor spheroids when tumor cells digested from tumor tissues of 4T1 tumor-bearing mice at the end of tumor growth inhibition experiments were seeded in soft 3D fibrin gels for 5 days. Data were represented as mean $\pm \mathrm{SD}(n=6)$. ${ }^{\star} P<0.05$, ${ }^{\star \star \star} P<0.001$ (one-way ANOVA with Bonferroni's multiple comparisons test for $\mathbf{a}, \mathbf{b}, \mathbf{d}, \mathbf{e}$ and log-rank test for c). Source data are provided as a Source Data file 
Furthermore, the mice model bearing B16-F10 melanoma with high lung metastasis was developed to evaluate the cross-reactive anticancer and CSCs killing activity of DOX@E-PSiNPs. At $48 \mathrm{~h}$ after injection of $5 \times 10^{5}$ B16-F10 cells into C57BL/6 mice, the mice were intravenously administrated with free DOX, DOX@PSiNPs, DOX@E-PSiNPs exocytosed from $\mathrm{H} 22$ cells at DOX dosage of $0.5 \mathrm{mg} \mathrm{kg}^{-1}$ or free DOX at high dosage of $4 \mathrm{mg} \mathrm{kg}^{-1}$ once every 3 days for 13 days. Significantly fewer metastatic nodules were detected in the DOX@E-PSiNPs-treated group (Fig. 9a and Supplementary Fig. 37). The less lung metastasis in DOX@E-PSiNPs-treated mice was further confirmed by H\&E staining on lungs (Fig. 9b). Mice treated with DOX@E-PSiNPs had 18,17 , and 4 days longer survival time as compared to free DOX and DOX@PSiNPs at DOX dosage of $0.5 \mathrm{mg} \mathrm{kg}^{-1}$, and free DOX at $4 \mathrm{mg} \mathrm{kg}^{-1}$ dosage (Fig. 9c). Furthermore, the fewest colony number and smallest colony size of the formed tumor spheroids were detected in DOX@E-PSiNPs-treated group after seeding the tumor cells digested from lungs in the $3 \mathrm{D}$ fibrin gels
(Fig. 9d, e). These results strongly demonstrate the excellent anticancer and CSCs killing efficacy of DOX@E-PSiNPs, regardless of tumor models used and the origin of exosomes used in DOX@E-PSiNPs. The cross-reactive anticancer treatment of DOX@E-PSiNPs did not induce immunological reaction, as evidenced by the fact that treatment with DOX@E-PSiNPs exocytosed from $\mathrm{H} 22$ cells did not affect the content of IgM, TNF- $\alpha$, IL-1 $\beta$, and IL- 6 in serum of C57BL/6 mice (Supplementary Fig. 38).

\section{Discussion}

CSCs, a small population of cancer cells with self-renewal and high tumorigenesis, play an important role in tumor development, progression and metastasis ${ }^{31,32}$. Traditional chemotherapeutics kill bulk tumor cells, but can not efficiently eliminate CSCs due to their overexpression of ATP-binding cassette $(\mathrm{ABC})$ transporters, antiapoptotic proteins and DNA repair enzymes, resulting in drug resistance and tumor recurrence after chemotherapy ${ }^{31,32}$. a
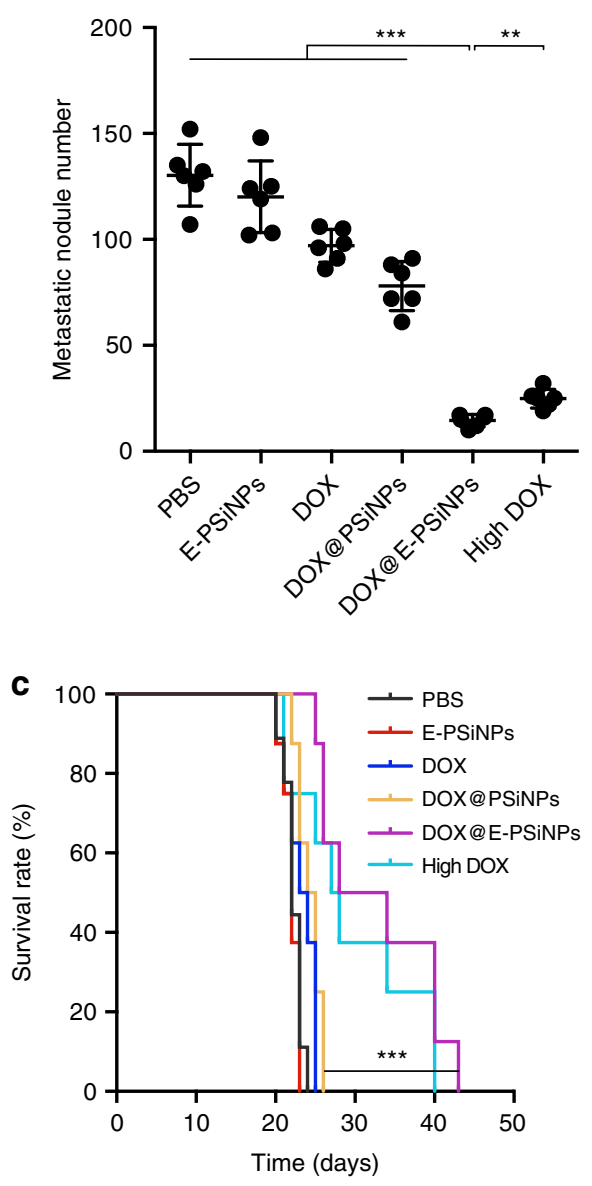

b

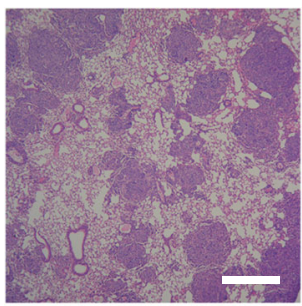

DOX@PSiNPs

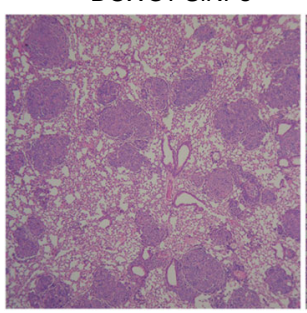

d

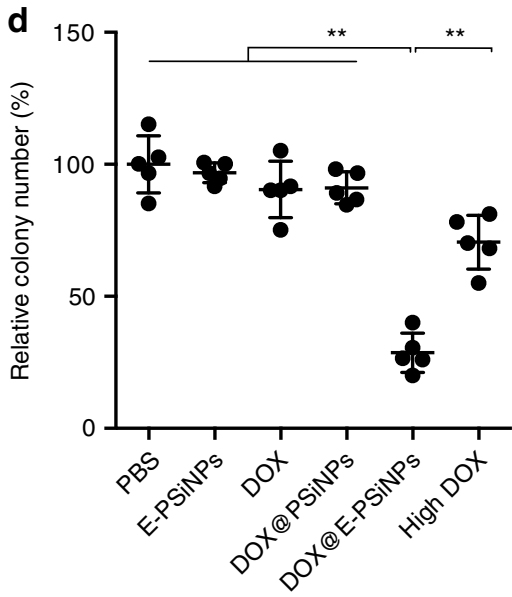

E-PSiNPs

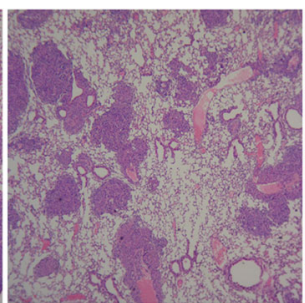

DOX@E-PSiNPs

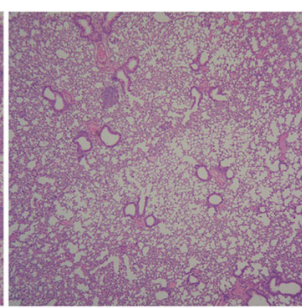

DOX

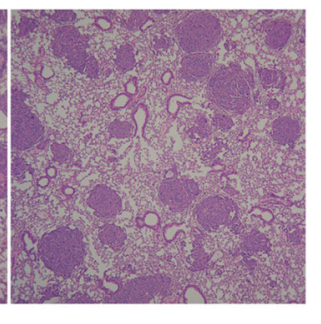

High DOX

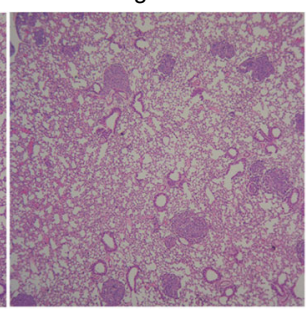

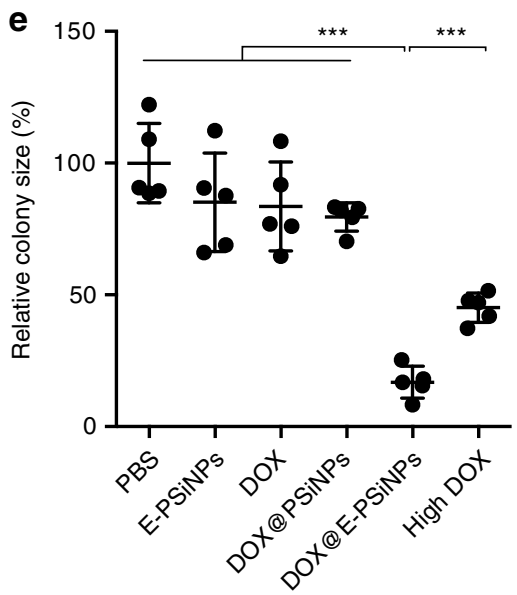

Fig. 9 Anticancer activity of DOX@E-PSiNPs in B16-F10 lung metastasis mice. a Metastatic nodule numbers in lungs of B16-F10 tumor-bearing mice after intravenous injection of PBS, E-PSiNPs, free DOX, DOX@PSiNPs, DOX@E-PSiNPs exocytosed from H22 cells at DOX dosage of 0.5 mg kg-1, or free DOX at high dosage of $4 \mathrm{mg} \mathrm{kg}^{-1}$ every three days for 13 days. Data were represented as mean $\pm \mathrm{SD}(n=6)$. $\mathbf{b}$ H\&E staining of lungs of B16-F10 tumor-bearing mice at the end of tumor growth inhibition experiments. Scale bar: $1000 \mu \mathrm{m}$. c Kaplan-Meier survival plot of B16-F10 tumor-bearing mice after intravenous administration of different formulations $(n=8)$. d, e Relative colony number $(\mathbf{d})$ and size $(\mathbf{e})$ of tumor spheroids when tumor cells digested from lung tumor nodules at the end of tumor growth inhibition experiments were seeded in soft 3D fibrin gels for 5 days. Data were represented as mean \pm SD ( $n=$ 5). ${ }^{\star \star} P<0.01,{ }^{\star \star \star} P<0.001$ (one-way ANOVA with Bonferroni's multiple comparisons test for $\mathbf{a}, \mathbf{d}, \mathbf{e}$ and log-rank test for $\mathbf{c}$ ). Source data are provided as a Source Data file 
Therefore, developing effective therapeutic strategies targeted to CSCs remains a big challenge for cancer therapy.

Nowadays, some NDDSs have been successfully applied to target CSCs to treat tumor. These approaches mainly include: (1) NDDSs were rationally designed to bypass the efflux pump via endocytosis, resulting in higher intracellular accumulation in $\mathrm{CSCs}^{46,47}$; (2) NDDSs codelivered MDR modulators and anticancer drugs to CSCs to overcome drug resistance ${ }^{48}$; and (3) NDDSs were modified with CSCs targeting ligands, such as $\mathrm{CD} 44^{49}, \mathrm{CD} 133^{50}$, and CD90 51 to increase specificity and cellular uptake. Although these NDDSs have shown potentials to overcome chemoresistance and enhance the accumulation of anticancer drug in CSCs, they cannot achieve full therapeutic efficacy. The main reasons lie in: (1) Ideal NDDSs targeting CSCs should be characterized by enhanced tumor accumulation, tumor penetration and cellular uptake by CSCs to highly enriched in CSCs following systemic administration ${ }^{52}$. However, the above approaches used to target CSCs are difficult to meet all demands at the same time, hindering the therapeutic efficacy; (2) There is no universal marker used for CSCs targeting in all cancers since the markers of CSCs differ from one type of tumor to another, and these markers are often expressed by other cell types, such as normal stem cells ${ }^{6,7}$. Thus, targeting NDDSs to CSCs using these markers is unreliable and risky; and (3) The constructed nanoparticles usually need complicated synthesis, and are usually toxic and may cause side effects as foreign components ${ }^{53}$. In the present study, we developed an exosome-sheathed PSiNPs to load DOX for efficient CSCs targeting and killing. DOX@E-PSiNPs not only exhibited enhanced tumor accumulation and penetration, but also had strong cross-reactive cellular uptake and cytotoxicity against CSCs, as evidenced by the fact that DOX@E-PSiNPs exocytosed from both $\mathrm{H} 22$ and B16-F10 cells are efficiently internalized into $\mathrm{H} 22$ and B16-F10 CSCs, resulting in the strongest cytotoxicity compared with free DOX and DOX@PSiNPs. The strong cross-reactive cellular uptake of DOX@E-PSiNPs can overcome the obstacles of requiring the specific markers for targeting CSCs in different tumors. Furthermore, DOX@EPSiNPs significantly decreased P-gp expression in CSCs, enhancing DOX retention in CSCs to overcome drug resistance. Therefore, DOX@E-PSiNPs efficiently integrated all features to eradicate CSCs, generating remarkable anticancer and CSCs killing activity in $\mathrm{H} 22$ tumor-bearing BALB/c mice, othotopic 4T1 tumor-bearing mice and B16-F10 tumor-bearing C57BL/6 mice. No significant toxicity of DOX@E-PSiNPs was observed in tumor-bearing mice by serological and histopathological analysis. Moreover, DOX@E-PSiNPs exocytosed from H22 cells, which were originated from liver cancer ascites of BALB/c mice, did not induce immune response in C57BL/6 mice, suggesting that DOX@E-PSiNPs are biocompatible and safe.

Upon autophagy induction, cytoplasmic materials are sequestered in double-membrane vesicles termed autophagosomes, which can fuse with MVBs to form amphisomes or directly deliver to the lysosomes for degradation ${ }^{35}$. Thus, the induction of autophagy usually inhibits the release of exosomes ${ }^{54}$. However, when the cells can not degrade material in the lysosomes due to the lysosomal defect, lysosomal overload or transport interference, the contents of lysosomes, MVBs or amphisomes are exocytosed as exosomes when fusing with cell membrane $e^{53}$. Several nanoparticles, such as silver nanoparticles ${ }^{55}$, carbonbased nanoparticles ${ }^{56}$ or silicon-based nanoparticles ${ }^{57}$, were reported to induce autophagy. In this work, E-PSiNPs used as an anticancer drug carrier, were exocytosed from cancer cells in an autophagy-dependent manner. The possible reason is due to the unique structure of PSiNPs, which cannot be degraded under lysosomal acidic microenvironment ${ }^{58}$ (Supplementary Fig. 39), promoting cancer cells to release exosome-coated PSiNPs. The exocytosed E-PSiNPs might keep the protein integrity on exosome membranes, which can display fully the biological function of exosomes during drug delivery.

In summary, we have successfully developed biocompatible exosome-sheathed PSiNPs for targeted cancer chemotherapy. DOX@E-PSiNPs are exocytosed from tumor cells after incubation with DOX@PSiNPs. Following intravenous injection, DOX@EPSiNPs exhibit enhanced tumor accumulation, tumor penetration and cross-reactive cellular uptake by bulk cancer cells and CSCs, resulting in augmented in vivo DOX enrichment in total tumor cells and side population cells. DOX@E-PSiNPs further demonstrate significant cross-reactive anticancer and CSCs killing activity in both subcutaneous transplantation tumor models, orthotopic tumor models and the advanced metastatic tumor models. Our study clearly demonstrates that exosome-biomimetic nanoparticles have potential as drug carriers to improve the anticancer efficacy.

\section{Methods}

Materials. Boron-doped p-type silicon wafers $(0.8-1.2 \mathrm{~m} \Omega \mathrm{cm}$ resistivity, $\langle 100\rangle$ orientation) were produced from Virginia Semiconductor, Inc. (Fredericksburg, VA, USA). Doxorubicin hydrochloride ( $\mathrm{DOX} \cdot \mathrm{HCl}$, with purity $>98.0 \%$ ) was obtained from Beijing HuaFeng United Technology CO., Ltd. (Beijing, China). RPMI 1640 medium, Dulbecco's Modified Eagle's Medium (DMEM), FBS, penicillin and streptomycin were provided by Gibco BRL/Life Technologies (Grand Island, NY, USA). Fibrinogen and thrombin were purchased from Searun Holdings Company (Freeport, ME, USA). Collagenase type I was purchased from Thermo Fisher Scientific (Waltham, MA, USA). Dispase II and TUNEL assay kit were purchased from F. Hoffmann-La Roche Ltd (Basel, Switzerland). Anti ICAM-1 antibody and anti P-gp antibody were purchased from ProteinTech (Wuhan, China). DIO, ionomycin, Hoechst 33342 and BCA protein quantification kit were purchased from Beyotime Biotechnology (Shanghai, China). Verapamil was provided by Selleck Chemicals (Houston, TX, USA). Cell counting kit (CCK-8) assay was obtained from Biosharp Company (Shanghai, China). DMA was purchased from Sigma-Aldrich (St Louis, MO, USA). All other reagents were of analytical grade and used without any further purification.

Cell lines and animals. Murine hepatocarcinoma cell line H22, mouse breast cancer cell line 4T1 and human hepatocarcinoma cell line Bel7402 were obtained from Type Culture Collection of Chinese Academy of Sciences (Shanghai, China) Murine melanoma cell line B16-F10 was kindly provided by Dr. Bo Huang (Huazhong University of Science and Technology, Wuhan, China). Wild type MEFs and Atg7 ${ }^{-/-}$MEFs were kindly provided by Dr. Mingzhou Chen (Wuhan University, Wuhan, China). H22 cells were cultured in RPMI 1640 medium, and Bel7402 cells, wild type and Atg7 $7^{-1-}$ MEFs and B16-F10 cells were cultured in DMEM medium at $37^{\circ} \mathrm{C}$ in a $5 \% \mathrm{CO}_{2}$ humidified incubator. All media contained $10 \% \mathrm{FBS}, 100 \mathrm{U} \mathrm{mL}^{-1}$ penicillin and $100 \mu \mathrm{g} \mathrm{mL}^{-1}$ streptomycin. Six- to eightweek-old $\mathrm{BALB} / \mathrm{c}$ mice (male and female) and C57BL/6 mice (male) were purchased from Beijing Vital River Laboratory Animal Technology Co., Ltd. (Beijing, China). H22 tumor-bearing mice were constructed by subcutaneously injecting $10^{6} \mathrm{H} 22$ cells per mouse into the flanks of male BALB/c mice. Orthotopic 4T1 breast tumor mode was constructed by injecting $2 \times 10^{5} 4 \mathrm{~T} 1$ cells to the right mammary fat pad of female BALB/c mice. B16-F10 lung metastasis tumor model was constructed by intravenously injecting $5 \times 10^{5}$ B16-F10 cells per mouse into C57BL/6 mice. All animal experiments comply with relevant ethical regulations for animal testing and research, and were approved by the Institutional Animal Care and Use Committee at Tongji Medical College, Huazhong University of Science and Technology (Wuhan, China). All cell lines were routinely tested for mycoplasma infection and were found to be negative by MycAway-Color one-step mycoplasma detection kit.

CSC culture. CSCs were selected by soft 3D fibrin gels ${ }^{42,43}$. Fibrinogen was diluted to $2 \mathrm{mg} \mathrm{mL}^{-1}$ with $\mathrm{T} 7$ buffer $(50 \mathrm{mM}$ Tris, $\mathrm{pH} 7.4,150 \mathrm{mM} \mathrm{NaCl})$ and then fibrinogen/cell mixtures were obtained by blending $2 \mathrm{mg} \mathrm{mL}^{-1}$ fibrinogen with similar volume of cell solution $\left(2 \times 10^{3}\right.$ cells per $\left.\mathrm{mL}\right)$, which produced gels of $90 \mathrm{~Pa}$ in elastic stiffness. $250 \mu \mathrm{L}$ mixtures were loaded into each well of 24 -well plate preadded with $5 \mu \mathrm{L}$ thrombin $\left(0.1 \mathrm{U}_{\mu} \mathrm{L}^{-1}\right)$. The cell culture plate was then incubated at $37^{\circ} \mathrm{C}$ for $30 \mathrm{~min}$. Finally, $1 \mathrm{~mL}$ RPMI 1640 medium containing $10 \%$ FBS and antibiotics were added. On the fifth day, tumor spheroids were obtained and digested into single cells using $0.08 \%$ collagenase type I and $0.4 \%$ dispase II for $20 \mathrm{~min}$ at $37^{\circ} \mathrm{C}$.

Preparation of PSiNPs and DOX@PSiNPs. PSiNPs were prepared by electrochemical etching method ${ }^{25-28}$. Briefly, boron-doped p-type silicon wafers were 
immersed into an aqueous solution of hydrofluoric acid (HF) and ethanol (4:1, v/v) in a Teflon etch cell, and then subjected to etch at a constant current density of $165 \mathrm{~mA} \mathrm{~cm}^{-2}$ for $300 \mathrm{~s}$. The rufous porous silicon film on the substrate was removed in 3.3\% aqueous HF solution in ethanol at a constant current of $4.5 \mathrm{~mA} \mathrm{~cm}^{-2}$ for $90 \mathrm{~s}$, fragmented in ultrapure water by ultrasonication overnight, and then centrifuged at $10,000 \mathrm{~g}$ for $20 \mathrm{~min}$ to collect PSiNPs. Finally, PSiNPs were heated at $60^{\circ} \mathrm{C}$ for $3 \mathrm{~h}$ to activate photoluminescence.

DOX@PSiNPs were prepared by adding PSiNPs in DOX solution at a weight ratio of 10:3 and then stirring for $12 \mathrm{~h}$ at room temperature. The mixtures were centrifuged at 10,000 g for $10 \mathrm{~min}$ to collect DOX@PSiNPs, followed by gently washing with ultrapure water twice to eliminate free DOX.

Autophagy induced by PSiNPs. H22 or Bel7402 cells were treated with $200 \mu \mathrm{g} \mathrm{mL}$ ${ }^{-1}$ PSiNPs for $6 \mathrm{~h}$. After washing with PBS for three times, cells were lysed in RIPA lysis buffer and then subjected to western blot analysis. Briefly, $200 \mu \mathrm{g}$ of lysates were separated by sodium dodecyl sulfate-polyacrylamide gel electrophoresis (SDSPAGE, $15 \%$ gel) and transferred onto nitrocellulose membranes. The membranes were blocked by $5 \%$ BSA for $2 \mathrm{~h}$, and then incubated with anti-LC3 (Novus, NB100-2331SS) and anti- $\beta$-actin antibody (Beyotime, AA128, diluted to $1: 2,000$ ) at $4{ }^{\circ} \mathrm{C}$ overnight. After washing with Tris-buffered saline containing $0.1 \%$ Tween-20 (TBST), the membranes were incubated with horseradish peroxidase (HRP) labeled secondary antibody (Beyotime, A0216, A0208, diluted to $1: 10,000$ ) at $37^{\circ} \mathrm{C}$ for $2 \mathrm{~h}$. The protein bands were detected using enhanced chemiluminescence (ECL) reagent and analyzed on ChemiDoc XRS Gel image system (Bio-Rad, Hercules, CA, USA). Uncropped gel images are provided in Source Data file.

Bel7402 cells were transfected with EGFP-LC3 plasmid by electroporation. After $24 \mathrm{~h}$ transfection, the cells were treated with $200 \mu \mathrm{g} \mathrm{mL}^{-1}$ PSiNPs for $6 \mathrm{~h}$, washed with PBS for three times and then fixed with $4 \%$ paraformaldehyde. Green fluorescence of LC3 proteins were visualized by FV1000 confocal microscope (Olympus, Japan).

Preparation and characterization of DOX@E-PSiNPs. To prepare E-PSiNPs or DOX@E-PSiNPs, $5 \times 10^{7} \mathrm{H} 22$, Bel7402, or B16-F10 cells were treated with PSiNPs (at silicon concentration of $200 \mu \mathrm{g} \mathrm{mL} \mathrm{L}^{-1}$ ) or DOX@PSiNPs (at DOX concentration of $10 \mu \mathrm{g} \mathrm{mL}^{-1}$ ) for $6 \mathrm{~h}$ in $10 \mathrm{~cm}$ dishes. Subsequently, the media were discarded and replaced with fresh one without PSiNPs or DOX@PSiNPs. After 16 h incubation, the debris was discarded at 5,000 $\mathrm{g}$ for $15 \mathrm{~min}$ and then the supernatants were further centrifuged at 20,000 g for 30 min to pellet out E-PSiNPs or DOX@EPSiNPs. Then, the obtained pellets were washed with PBS and resuspended in PBS for further experiments. DOX loading into E-PSiNPs was confirmed by labeling DOX@E-PSiNPs with DiO and then observed by FV1000 confocal microscopy. The $\mathrm{DiO}$ fluorescence was detected at the excitation wavelength of $488 \mathrm{~nm}$ and the emission range of $500-520 \mathrm{~nm}$, DOX at the excitation wavelength of $559 \mathrm{~nm}$ and the emission range of 570-600 nm, and PSiNPs at the excitation wavelength of 488 and the emission range of $670-690 \mathrm{~nm}$. The hydrodynamic diameter of E-PSiNPs and DOX@E-PSiNPs was determined by DLS (ZetaSizer ZS90, Malvern Instruments Ltd., Worcestershire, UK). The morphology of E-PSiNPs was observed by TEM (Tecnai G2-20, FEI Corp., Netherlands). DOX content loaded into DOX@EPSiNPs was determined by incubating in $1 \mathrm{M} \mathrm{NaOH}$ for 30 min to dissolve $\mathrm{E}-$ PSiNPs, neutralizing with equal volume of $1 \mathrm{M} \mathrm{HCl}$ and then detecting DOX content by HPLC.

Exosome purification. Exosomes were purified using differential ultracentrifugation $\operatorname{method~}^{37,38}$. First, FBS used for cell incubation was centrifuged at $100,000 \mathrm{~g}$ overnight to wipe out the existing exosomes. $\mathrm{H} 22$ or Bel7402 cells were incubated in exosome-free RPMI 1640 or DMEM medium for $48 \mathrm{~h}$. Cell culture medium was collected and sequentially centrifuged at $1000 \mathrm{~g}$ for $10 \mathrm{~min}, 10,000 \mathrm{~g}$ for $30 \mathrm{~min}$ and $100,000 \mathrm{~g}$ for $1 \mathrm{~h}$ to pellet exosomes. Exosomes were washed with PBS and recovered by centrifugation at $100,000 \mathrm{~g}$ for $1 \mathrm{~h}$.

Confirmation of exosomes sheathed on PSiNPs in E-PSiNPs. E-PSiNPs were stained with $10 \mu \mathrm{M} \mathrm{DiO}$ for $30 \mathrm{~min}$, centrifuged at $20,000 \mathrm{~g}$ for $30 \mathrm{~min}$ and then washed with PBS three times. The colocalization of DiO and PSiNPs was observed by FV1000 confocal microscopy. The fluorescence of $\mathrm{DiO}$ at $500-520 \mathrm{~nm}$ and PSiNPs at $670-690 \mathrm{~nm}$ was detected at the excitation of $488 \mathrm{~nm}$

E-PSiNPs was blocked by $5 \%$ BSA for $30 \mathrm{~min}$, and then incubated with FITCconjugated CD63 antibody (Biolegend, 353005, diluted to 1:200) for $30 \mathrm{~min}$ at room temperature. The colocalization of $\mathrm{CD} 63$ and PSiNPs was observed by FV1000 confocal microscopy. The fluorescence of FITC at 500-520 nm and PSiNPs at $670-690 \mathrm{~nm}$ was detected at the excitation of $488 \mathrm{~nm}$.

Whole cells, the purified exosomes and E-PSiNPs were lysed in RIPA lysis buffer and then subjected to western blot analysis. The primary antibodies used included anti-CD63 (Abcam, ab216130), anti-TSG101 (Santa Cruz, SC-7964) and anti-calnexin (Beyotime, AC018). All primary antibodies were diluted to 1:2000. Uncropped gel images are provided in Source Data file.

E-PSiNPs yield. Bel7402 cells were incubated with $200 \mu \mathrm{g} \mathrm{mL}-1$ PSiNPs for $6 \mathrm{~h}$ and washed with PBS three times. Then fresh medium containing $200 \mathrm{nM}$ rapamycin, $30 \mu \mathrm{M} \mathrm{CBZ,} 5 \mathrm{mM}$ 3-MA, $15 \mathrm{nM}$ DMA or $10 \mu \mathrm{M}$ ionomycin were added. After $16 \mathrm{~h}$ incubation, the supernatants were collected, centrifuged at $5000 \mathrm{~g}$ for $15 \mathrm{~min}$ to remove debris, and then centrifuged at $20,000 \mathrm{~g}$ for $30 \mathrm{~min}$. The pellets were dissolved in $1 \mathrm{M} \mathrm{NaOH}$ solution and silicon content was measured by Optima 4300 DV ICP-OES (PerkinElmer, Norwalk, CT, USA).

In vitro DOX release profile. DOX release profile from DOX@E-PSiNPs was determined by dialysis method. Briefly, DOX@E-PSiNPs (300 $\mu \mathrm{g}$ DOX content) were put into a dialysis bag (cutoff molecular weight was $3000 \mathrm{Da}$ ) and submerged fully into PBS $(30 \mathrm{~mL})$, then stirred with $250 \mathrm{rpm}$ at $37^{\circ} \mathrm{C}$. At the designated time intervals, $0.5 \mathrm{~mL}$ of sample solution was taken out and replaced with equal amount of fresh PBS. DOX content in samples was measured by HPLC.

Interaction between DOX@E-PSiNPs and CSCs by AFM. H22 CSCs were seeded on coverslips pretreated with poly-lysine in 6-well plates at a density of $3 \times 10^{5}$ cells per well. H22 CSCs were then incubated with DOX, DOX@PSiNPs or DOX@EPSiNPs at the DOX concentration of $2 \mu \mathrm{g} \mathrm{mL}-1$ at $37^{\circ} \mathrm{C}$ for $2 \mathrm{~h}$. After washing with PBS, CSCs were fixed with $0.25 \%$ (v/v) glutaraldehyde for $30 \mathrm{~min}$ at room temperature. The coverslips were rinsed with deionized water to remove salt crystals and air dried before analysis. AFM images were obtained using a multimode 8 AFM (Bruker, Santa Barbara, CA, USA). Cell surface studies were performed in ScanAsys mode at scan frequencies below $1 \mathrm{~Hz}$. The roughness of CSCs membrane was analyzed by measurement of Image Rq.

Cell membrane fluidity. H22 CSCs membrane fluidity was measured using fluorescence polarization of 1,6-diphenyl-1,3,5-hexatriene (DPH) ${ }^{59}$. Briefly, $\mathrm{H} 22$ CSCs $\left(10^{6}\right.$ cell per mL) were incubated with DPH $(2 \mu \mathrm{M})$ at $37^{\circ} \mathrm{C}$ for $1 \mathrm{~h}$. The labeled CSCs were then incubated with DOX, DOX@PSiNPs or DOX@E-PSiNPs at the DOX concentration of $2 \mu \mathrm{g} \mathrm{mL}^{-1}$ for $2 \mathrm{~h}$. Fluorescence anisotropy was measured using a polarization spectrofluorometer (FP-6500, Jasco, Tokyo, Japan) with an excitation wavelength of $365 \mathrm{~nm}$ and an emission wavelength of $429 \mathrm{~nm}$. Anisotropy was calculated as: $r=\left(\mathrm{I}_{\mathrm{VV}}-\mathrm{I}_{\mathrm{VH}} \cdot \mathrm{G}\right) /\left(\mathrm{I}_{\mathrm{VV}}+\mathrm{I}_{\mathrm{VH}} \cdot \mathrm{G}\right)$, where $G=\mathrm{I}_{\mathrm{HV}} / \mathrm{I}_{\mathrm{HH}}$ is used to correct the unequal transmission of the optics.

Internalization into bulk cancer cells and CSCs. Bel7402 and H22 cells, and $\mathrm{H} 22$ and B16-F10 CSCs selected in soft 3D fibrin gels were seeded into six-well plates overnight at a density of $2 \times 10^{5}$ cells per well. Subsequently, cells were incubated with free DOX, DOX@PSiNPs or DOX@E-PSiNPs at different DOX concentrations for $2 \mathrm{~h}$, rinsed with PBS and then collected to analyze the intracellular DOX fluorescence in FL2 channel by flow cytometry (FC500, Beckman Coulter, Fullerton, CA, USA)

Cytotoxicity against bulk cancer cells and CSCs. For determination of DOX@EPSiNPs against bulk cancer cells, Bel7402, H22 and B16-F10 cells were seeded in 96-well plate at a density of $8 \times 10^{3}$ cells per well overnight and then treated with free DOX, DOX@PSiNPs or DOX@E-PSiNPs at different DOX concentrations. After $24 \mathrm{~h}$ treatment, cell survival rate was detected by CCK- 8 assay.

To evaluate cytotoxicity of DOX@E-PSiNPs against CSCs, H22 and B16-F10 cells were pre-treated with DOX, DOX@PSiNPs or DOX@E-PSiNPs at different DOX concentrations for $4 \mathrm{~h}$. The cells were harvested, washed and counted. $4 \times 10^{2}$ cells from different groups were then seeded in 3D fibrin gels. On the fifth day, the numbers of tumor spheroids in different groups were counted under Olympus IX 71 optical microscope (Tokyo, Japan). Tumor spheroids in each group were imaged and their sizes were calculated by Image J software.

To further determine cytotoxicity of DOX@E-PSiNPs against CSCs, H22 and B16-F10 CSCs were selected in 3D fibrin gels in 96-well plates. On day 5, the media were aspirated and fresh media containing DOX, DOX@PSiNPs or DOX@EPSiNPs at DOX concentration of $2 \mu \mathrm{g} \mathrm{mL}-1$ were added. After $24 \mathrm{~h}$ incubation, tumor spheroids with integral rims in each group were counted under optical microscope. The images of tumor spheroids in each group were captured and their sizes were calculated by Image J software.

In vivo biodistribution. When tumor volume of $\mathrm{H} 22$ tumor-bearing mice reached ca. $250 \mathrm{~mm}^{3}$, or at 13 days after intravenous injection of B16-F10 cells into C57BL/ 6 mice, the mice were intravenously injected with free DOX, DOX@PSiNPs or DOX@E-PSiNPs exocytosed from H22 cells at DOX dosage of $0.5 \mathrm{mg} \mathrm{kg}^{-1}$, or free DOX at high dosage of $4 \mathrm{mg} \mathrm{kg}^{-1}$. At $24 \mathrm{~h}$ post-injection, the mice were sacrificed and the major organs (heart, liver, spleen, lung and kidney) and tumors in H22 tumor-bearing mice and lung metastatic nodules in B16-F10 tumor-bearing mice were collected. Subsequently, tissues were lysed and DOX was extracted by incubating the lysates in $1 \mathrm{M} \mathrm{NaOH}$ for $30 \mathrm{~min}$ and neutralizing by the same volume of $1 \mathrm{M} \mathrm{HCl}$. DOX contents in the lysates were tested by a FlexStation3 Multi-Mode Microplate Reader (Molecular Devices, Sunnyvale, CA, USA).

In vitro penetration in 3D tumor spheroids. $\mathrm{H} 22$ tumor spheroids were constructed using soft 3D fibrin gel method as described above. To observe penetration of DOX@E-PSiNPs from outside of tumor spheroids to core area, tumor spheroids with diameter of ca. 150-200 $\mu \mathrm{m}$ were treated with free DOX, DOX@PSiNPs or 
DOX@E-PSiNPs exocytosed from H22 cells at DOX concentration of $2 \mu \mathrm{g} \mathrm{mL}$ for $24 \mathrm{~h}$. The spheroids were washed with PBS twice, fixed with $4 \%$ paraformaldehyde for $30 \mathrm{~min}$, and then transferred to confocal dishes. DOX red fluorescence at $570-590 \mathrm{~nm}$ was observed by confocal microscopy using Z-stack scanning mode at the intervals of $5 \mu \mathrm{m}$ at the excitation of $559 \mathrm{~nm}$. The 3D DOX fluorescence images in tumor spheroids were reconstructed by using Amira software.

In vivo tumor penetration. When tumor volume of $\mathrm{H} 22$ tumor-bearing mice reached ca. $250 \mathrm{~mm}^{3}$, the mice were intravenously injected with DOX, DOX@PSiNPs or DOX@E-PSiNPs exocytosed from H22 cells at DOX dosage of $0.5 \mathrm{mg} \mathrm{kg}$ ${ }^{-1}$. At $24 \mathrm{~h}$ post-injection, tumor tissues were collected, washed with PBS, and then frozen-sectioned into pieces. The sections were incubated with FITC-CD31 antibody (Biolegend, 102405, diluted to $1: 200$ ) at $37^{\circ} \mathrm{C}$ for $30 \mathrm{~min}$ to label tumor vessels, and then rinsed with PBS. DOX red fluorescence at $570-600 \mathrm{~nm}$ and FITC-CD31 green fluorescence at $500-520 \mathrm{~nm}$ were observed by confocal microscopy at the excitation of $559 \mathrm{~nm}$ or $488 \mathrm{~nm}$, respectively. DOX distribution from blood vessels to deep tumor tissues was measured by Image J Software.

Intercellular delivery. $\mathrm{H} 22$ cells were seeded on coverslips, which were pretreated with $10 \mu \mathrm{g} \mathrm{mL}^{-1}$ poly-lysine overnight. Cells on the first coverslip were treated with $2 \mu \mathrm{g} \mathrm{mL} \mathrm{m}^{-1}$ DOX, DOX@PSiNPs or DOX@E-PSiNPs for $6 \mathrm{~h}$. The treated cells were rinsed with PBS and then co-incubated with the new cells on the second coverslip for $16 \mathrm{~h}$ in fresh medium. Finally, the cells on the second coverslip were co-incubated with the new cells on the third coverslip for another $16 \mathrm{~h}$ in fresh medium. Cells were rinsed with PBS and the intercellular DOX fluorescence at $570-600 \mathrm{~nm}$ were analyzed by confocal microscope at the excitation of $559 \mathrm{~nm}$ and flow cytometry (FL2 channel).

In vivo DOX accumulation in total tumor cells and CSCs. When tumor volume of GFP-expressing $\mathrm{H} 22$ tumor-bearing mice reached ca. $250 \mathrm{~mm}^{3}$, the mice were intravenously injected with free DOX, DOX@PSiNPs or DOX@E-PSiNPs exocytosed from $\mathrm{H} 22$ cells at DOX dosage of $0.5 \mathrm{mg} \mathrm{kg}^{-1}$, or free DOX at high dosage of $4 \mathrm{mg} \mathrm{kg}^{-1}$. At $24 \mathrm{~h}$ after injection, tumor tissues were collected, washed with PBS and then cut into small pieces, followed by digestion with $1 \mathrm{mg} \mathrm{mL}^{-1}$ collagenase type I solution at $37^{\circ} \mathrm{C}$ for $2 \mathrm{~h}$. The single tumor cells were acquired by filtering the digested cells with 200-mesh nylon twice and then divided into two parts. One part was used to determine DOX content in total GFP-positive tumor cells by flow cytometry in FL2 channel. The other one was applied to determine DOX content in side population cells of GFP-positive tumor cells. The tumor cells were treated with $5 \mu \mathrm{g} \mathrm{mL}{ }^{-1}$ Hoechst 33342 for $90 \mathrm{~min}$ in the presence or absence of $50 \mu \mathrm{m}$ verapamil at $37^{\circ} \mathrm{C}$ in the dark. Living cells were plotted on SSC-A and FSC-A graph. Hoechst 33342 fluorescence at $450 \mathrm{~nm}$ or $660 \mathrm{~nm}$ in living GFP-positive tumor cells was measured. The gating of side population cells was plotted as the absence of cell population in PBS-treated group comparted with verapamil-treated group. DOX fluorescence intensity in side population cells was detected by flow cytometry (FL2 channel).

Anticancer activity in subcutaneous H22 tumor-bearing mice. When tumor volume of $\mathrm{H} 22$ tumor-bearing mice reached ca. $200 \mathrm{~mm}^{3}$, the mice were intravenously injected with PBS, E-PSiNPs, free DOX, DOX@PSiNPs, DOX@E-PSiNPs exocytosed from $\mathrm{H} 22$ cells at DOX dosage of $0.5 \mathrm{mg} \mathrm{kg}^{-1}$, or free DOX at high dosage of $4 \mathrm{mg} \mathrm{kg}^{-1}$ once every three days ( $n=14$ per group). The tumor sizes were measured every day via vernier caliper and the body weights of mice were also recorded. On 17th day of treatment, mice were further divided into two groups. One group $(n=8)$ was used for survival experiment, while the other part $(n=6)$ was used to estimate anticancer efficacy. For anticancer efficacy analysis, mice were sacrificed, and tumors and major organs (heart, liver, spleen, lung and kidney) were obtained and washed with PBS. The cleaned tumors were weighed and fixed with $4 \%$ paraformaldehyde, then sectioned and stained using a TUNEL assay kit according to the manufacturer's protocol. The major organs were also fixed with $4 \%$ paraformaldehyde, sectioned and examined by H\&E staining.

To estimate CSCs killing activity of DOX@E-PSiNPs in vivo, tumor tissues were digested into single cells after treatment as above using $1 \mathrm{mg} \mathrm{mL}^{-1}$ collagenase type I solution. One part of cells were used to determine the number of CD133-positive cells by flow cytometry. The second part of cells were seeded in soft 3D fibrin gels ( 400 cells per well) and incubated for 5 days ${ }^{42,43}$. The numbers of tumor spheroids were counted under optical microscope. The images of tumor spheroids were captured and their sizes were calculated by Image J software. The third part of cells were subcutaneously transplanted into BALB/c mice $\left(10^{6}\right.$ cells per mouse $)$ and the tumor formation ratio was evaluated. Furthermore, the anticancer treatment was performed in the GFP-expressing H22-tumor bearing mice as above. On 17th day of treatment, the mice were sacrificed and tumor tissues were digested into single cells. The cells were dyed with $5 \mu \mathrm{g} \mathrm{mL}{ }^{-1}$ Hoechst 33342 for $90 \mathrm{~min}$ at $37^{\circ} \mathrm{C}$ in the presence or absence of $50 \mu \mathrm{M}$ verapamil in the dark, then washed and resuspended in PBS. The number of side population cells in GFP-positive tumor cells was measured by flow cytometry (450/45 BP filter for blue fluorescence and 660/20 BP filter for red fluorescence).
Anticancer activity in orthotopic $4 \mathrm{T1}$ breast cancer mode. $4 \mathrm{~T} 1$ cells $\left(2 \times 10^{5}\right.$ cells) were suspended in $50 \mu \mathrm{L}$ PBS and then injected into the right forth breast fat pad. When the tumor volume reached $50-70 \mathrm{~mm}^{3}$, the mice were administrated with PBS, E-PSiNPs, free DOX, DOX@PSiNPs or DOX@E-PSiNPs at DOX dosage of $0.5 \mathrm{mg} \mathrm{kg}^{-1}$, or high dosage of DOX at $4 \mathrm{mg} \mathrm{kg}^{-1}$ once every three days for five times ( $n=14$ per group). The tumor sizes were measured every day via vernier caliper. On 15th day of treatment, mice were further divided into two groups. One group $(n=8)$ was used for survival experiment, while the other part $(n=6)$ was used to estimate anticancer efficacy.

To investigate CSCs killing activity of DOX@E-PSiNPs in vivo, tumor tissues were collected and digested into single cells using $1 \mathrm{mg} \mathrm{mL}^{-1}$ collagenase type I solution. The cells (400 tumor cells per well) were seeded in soft 3D fibrin gels. On day 5 , the numbers of tumor spheroids were counted under optical microscope. The images of tumor spheroids were captured and their sizes were calculated by Image J software.

Anticancer activity in B16-F10 lung metastasis cancer model. At $48 \mathrm{~h}$ after B16F10 cells $\left(5 \times 10^{5}\right.$ cells per mouse) were intravenously injected into C57BL/ 6 mice, the mice were intravenously administrated with PBS, E-PSiNPs, free DOX, DOX@PSiNPs, DOX@E-PSiNPs exocytosed from H22 cells at DOX dosage of $0.5 \mathrm{mg} \mathrm{kg}^{-1}$, or free DOX at high dosage of $4 \mathrm{mg} \mathrm{kg}^{-1}$ once every three days $(n=$ 14 per group). On 13th day of treatment, mice were divided into two parts. One part $(\mathrm{n}=8)$ was used for long-term survival experiment and the other part $(n=6)$ was used for evaluation of anticancer effect. For evaluation of anticancer effect, the mice were sacrificed and the lungs were acquired. The numbers of tumor nodules on the surface of lungs were recorded. Lungs were then fixed with $4 \%$ paraformaldehyde, sectioned and examined by $\mathrm{H} \& \mathrm{E}$ staining.

To investigate CSCs killing activity of DOX@E-PSiNPs in vivo, tumor nodules were collected and digested into single cells using $1 \mathrm{mg} \mathrm{mL}^{-1}$ collagenase type I solution. The cells (400 tumor cells per well) were seeded in soft 3D fibrin gels. On day 5 , the numbers of tumor spheroids were counted under optical microscope. The images of tumor spheroids were captured and their sizes were calculated by Image J software.

Immune response. C57BL/6 mice were intravenously injected with PBS, EPSiNPs, DOX, DOX@PSiNPs, DOX@E-PSiNPs exocytosed from H22 cells at the DOX dosage of $0.5 \mathrm{mg} \mathrm{kg}^{-1}$, or free DOX at high dosage of $4 \mathrm{mg} \mathrm{kg}^{-1}$. At different time intervals, the orbital blood was obtained, maintained for $30 \mathrm{~min}$ and centrifuged at $10,000 \mathrm{~g}$ for $10 \mathrm{~min}$. The serum was collected and the contents of IgM, IL-1 $\beta$, IL- 6 , and TNF- $\alpha$ were analyzed by enzyme linked immunosorbent assay (ELISA).

Statistical analysis. Experiments were performed with at least three replicates. All values were presented as mean values $\pm \mathrm{SD}$. Statistical analyses were carried out using the GraphPad Prism software version 6.0. Comparison between two groups was performed using unpaired two-tailed Student's $t$ test. One-way ANOVA or two-way ANOVA was used for comparison of more than two groups. Statistical significance for survival curves was determined using a log-rank test. Values with $P$ $<0.05$ are considered significant.

Reporting summary. Further information on research design is available in the Nature Research Reporting Summary linked to this article.

\section{Data availability}

The authors declare that the main data supporting the findings of this study are available within the article and its Supplementary Information. Extra data are available from the corresponding author upon reasonable request. The source data underlying Figs. 2-9 and Supplementary Figs. 1-39 are provided with the paper as a Source Data file. A reporting summary for this article is available as a Supplementary Information file.

Received: 9 February 2018 Accepted: 29 July 2019

Published online: 23 August 2019

\section{References}

1. Peer, D. et al. Nanocarriers as an emerging platform for cancer therapy. Nat. Nanotech. 2, 751-760 (2007).

2. Maeda, H., Nakamura, H. \& Fang, J. The EPR effect for macromolecular drug delivery to solid tumors: improvement of tumor uptake, lowering of systemic toxicity, and distinct tumor imaging in vivo. Adv. Drug Deliv. Rev. 65, 71-79 (2013)

3. Mitragotri, S., Burke, P. A. \& Langer, R. Overcoming the challenges in administering biopharmaceuticals: formulation and delivery strategies. Nat. Rev. Drug Discov. 13, 655-672 (2014). 
4. Cheng, C. J. et al. A holistic approach to targeting disease with polymeric nanoparticles. Nat. Rev. Drug Discov. 14, 239-247 (2015).

5. Salvati, A. et al. Transferrin-functionalized nanoparticles lose their targeting capabilities when a biomolecule corona adsorbs on the surface. Nat. Nanotechnol. 8, 137-143 (2013).

6. Magee, J. A., Piskounova, E. \& Morrison, S. J. Cancer stem cells: impact, heterogeneity, and uncertainty. Cancer Cell 21, 283-296 (2012)

7. Zhao, Y., Alakhova, D. Y. \& Kabanov, A. V. Can nanomedicines kill cancer stem cells? Adv. Drug Deliv. Rev. 65, 1763-1783 (2013).

8. Tan, S. et al. Cell or cell membrane-based drug delivery systems. Theranostics 5, 863-881 (2015)

9. Luk, B. T. \& Zhang, L. Cell membrane-camouflaged nanoparticles for drug delivery. J. Control. Release 220, 600-607 (2015).

10. Gao, W. et al. Surface functionalization of gold nanoparticles with red blood cell membranes. Adv. Mater. 25, 3549-3553 (2013).

11. Piao, J. G. et al. Erythrocyte membrane is an alternative coating to polyethylene glycol for prolonging the circulation lifetime of gold nanocages for photothermal therapy. ACS Nano 8, 10414-10425 (2014).

12. Chen, Z. et al. Cancer cell membrane-biomimetic nanoparticles for homologous-targeting dual-modal imaging and photothermal therapy. ACS Nano 10, 10049-10057 (2016).

13. Sun., H. et al. Cancer-cell-biomimetic nanoparticles for targeted therapy of homotypic tumors. Adv. Mater. 28, 9581-9588 (2016).

14. Wei., X. et al. Nanoparticles camouflaged in platelet membrane coating as an antibody decoy for the treatment of immune thrombocytopenia. Biomaterials 111, 116-123 (2016).

15. Parodi, A. et al. Synthetic nanoparticles functionalized with biomimetic leukocyte membranes possess cell-like functions. Nat. Nanotechnol. 8, 61-68 (2013).

16. Thery, C., Zitvogel, L. \& Amigorena, S. Exosomes: composition, biogenesis and function. Nat. Rev. Immunol. 2, 569-579 (2002).

17. Batrakova, E. V. \& Kim, M. S. Using exosomes, naturally-equipped nanocarriers, for drug delivery. J. Control. Release 219, 396-405 (2015)

18. van den Boorn, J. G. et al. Exosomes as nucleic acid nanocarriers. Adv. Drug Deliv. Rev. 65, 331-335 (2013).

19. Vader, P. et al. Extracellular vesicles for drug delivery. Adv. Drug Deliv. Rev. 106, 148-156 (2016)

20. Frydrychowicz, M. et al. Exosomes-structure, biogenesis and biological role in non-small-cell lung cancer. Scand. J. Immunol. 81, 2-10 (2015).

21. Jang, S. C. et al. Bioinspired exosome-mimetic nanovesicles for targeted delivery of chemotherapeutics to malignant tumors. ACS Nano 7, 7698-7710 (2013).

22. Sato, Y. T. et al. Engineering hybrid exosomes by membrane fusion with liposomes. Sci. Rep. 6, 21933 (2016).

23. Fuhrmann, G. et al. Active loading into extracellular vesicles significantly improves the cellular uptake and photodynamic effect of porphyrins. $J$. Control. Release 205, 35-44 (2015).

24. Haney, M. J. et al. Exosomes as drug delivery vehicles for Parkinson's disease therapy. J. Control. Release 207, 18-30 (2015).

25. Park, J. H. et al. Biodegradable luminescent porous silicon nanoparticles for in vivo applications. Nat. Mater. 8, 331-836 (2009).

26. Yong, T. et al. Domino-like intercellular delivery of undecylenic acidconjugated porous silicon nanoparticles for deep tumor penetration. ACS Appl. Mater. Interfaces 8, 27611-27621 (2016).

27. Fontana, F. et al. Multistaged nanovaccines based on porous silicon@acetalated dextran@cancer cell membrane for cancer immunotherapy. Adv. Mater. 29, 1603239 (2017).

28. $\mathrm{Xu}, \mathrm{R}$. et al. An injectable nanoparticle generator enhances delivery of cancer therapeutics. Nat. Biotechnol. 34, 414-418 (2016).

29. Wareing, N. et al. In vitro gene delivery with large porous silicon nanoparticles fabricated using cost-effective, metal-assisted chemical etching. Small 13, 1602739 (2017).

30. $\mathrm{Gu}, \mathrm{L}$. et al. Multivalent porous silicon nanoparticles enhance the immune activation potency of agonistic CD40 antibody. Adv. Mater. 24, 3981-3987 (2012).

31. Visvader, J. E. \& Lindeman, G. J. Cancer stem cells in solid tumours: accumulating evidence and unresolved questions. Nat. Rev. Cancer 8, 755-768 (2008).

32. Beck, B. \& Blanpain, C. Unravelling cancer stem cell potential. Nat. Rev. Cancer 13, 727-738 (2013).

33. Ho, M. M. et al. Side population in human lung cancer cell lines and tumors is enriched with stem-like cancer cells. Cancer Res. 67, 4827-4833 (2007).

34. Nakanishi, T. et al. Side-population cells in luminal-type breast cancer have tumour-initiating cell properties, and are regulated by HER 2 expression and signalling. Br. J. Cancer 102, 815-826 (2010).

35. Levine, B., Mizushima, N. \& Virgin, H. W. Autophagy in immunity and inflammation. Nature 469, 323-335 (2011).
36. Kabeya, Y. et al. LC3, a mammalian homologue of yeast Apg8p, is localized in autophagosome membranes after processing. EMBO J. 19, 5720-5728 (2000)

37. Rao, Q. et al. Tumor-derived exosomes elicit tumor suppression in murine hepatocellular carcinoma models and humans in vitro. Hepatology 64, 456-472 (2016).

38. Saari, H. et al. Microvesicle- and exosome-mediated drug delivery enhances the cytotoxicity of Paclitaxel in autologous prostate cancer cells. J. Control. Release 220, 727-737 (2015).

39. Lobb, R. J. et al. Optimized exosome isolation protocol for cell culture supernatant and human plasma. J. Extracell. Vesicles 4, 27031 (2015).

40. Miao, Y. et al. A TRP channel senses lysosome neutralization by pathogens to trigger their expulsion. Cell 161, 1306-1319 (2015).

41. Hoffman, E. A. et al. Regulation of myocilin-associated exosome release from human trabecular meshwork cells. Invest. Ophthalmol. Vis. Sci. 50, 1313-1318 (2009).

42. Liu, J. et al. Soft fibrin gels promote selection and growth of tumorigenic cells. Nat. Mater. 11, 734-741 (2012).

43. Ma., J. et al. Reversing drug resistance of soft tumor-repopulating cells by tumor cell-derived chemotherapeutic microparticles. Cell Res. 26, 713-727 (2016).

44. Peetla, C., Vijayaraghavalu, S. \& Labhasetwar, V. Biophysics of cell membrane lipids in cancer drug resistance: implications for drug transport and drug delivery with nanoparticles. Adv. Drug Deliv. Rev. 65, 1686-1698 (2013).

45. Clevers, $\mathrm{H}$. The cancer stem cell: premises, promises and challenges. Nat. Med. 17, 313-319 (2011)

46. Wang., X. et al. Epirubicin-adsorbed nanodiamonds kill chemoresistant hepatic cancer stem cells. ACS Nano 8, 12151-12166 (2014).

47. Rao, W. et al. Chitosan-decorated doxorubicin-encapsulated nanoparticle targets and eliminates tumor reinitiating cancer stem-like cells. ACS Nano $\mathbf{9}$, 5725-5740 (2015).

48. Liu, J. et al. A tailored DNA nanoplatform for synergistic RNAi/chemotherapy of multidrug-resistant tumors. Angew. Chem. Int. Ed. Engl. 57, 15486-15490 (2018).

49. Shen, H. et al. Coating solid lipid nanoparticles with hyaluronic acid enhances antitumor activity against melanoma stem-like cells. Theranostics $\mathbf{5}, \mathbf{7 5 5 - 7 7 1}$ (2015).

50. Ning, S. T. et al. Targeting colorectal cancer stem-like cells with anti-CD133 antibody-conjugated SN-38 nanoparticles. ACS Appl. Mater. Interfaces 8 17793-17804 (2016).

51. Yang, Z. F. et al. Significance of $\mathrm{CD}^{+} 0^{+}$cancer stem cells in human liver cancer. Cancer Cell 13, 153-166 (2008).

52. Shen, S., Xia, J. X. \& Wang, J. Nanomedicine-mediated cancer stem cell therapy. Biomaterials 74, 1-18 (2016).

53. Fang, R. H. et al. Engineered nanoparticles mimicking cell membranes for toxin neutralization. Adv. Drug Deliv. Rev. 90, 69-80 (2015).

54. Hessvik, N. P. \& Llorente, A. Current knowledge on exosome biogenesis and release. Cell. Mol. Life Sci. 75, 193-208 (2018).

55. Lin, J. et al. Inhibition of autophagy enhances the anticancer activity of silver nanoparticles. Autophagy 10, 2006-2020 (2014)

56. $\mathrm{Wu}$, L. et al. Tuning cell autophagy by diversifying carbon nanotube surface chemistry. ACS Nano 8, 2087-2099 (2014).

57. Ha, S. W., Weitzmann, M. N. \& Beck, G. R. Jr. Bioactive silica nanoparticles promote osteoblast differentiation through stimulation of autophagy and direct association with LC3 and p62. ACS Nano 8, 5898-5910 (2014).

58. Jarvis, K. L., Barnes, T. J. \& Prestidge, C. A. Surface chemistry of porous silicon and implications for drug encapsulation and delivery applications. Adv. Colloid Interface Sci. 175, 25-38 (2012).

59. Xiao, L. et al. Role of cellular uptake in the reversal of multidrug resistance by PEG-b-PLA polymeric micelles. Biomaterials 32, 5148-5157 (2011).

\section{Acknowledgements}

This work was supported by National Basic Research Program of China (2018YFA0208900 and 2015CB931800), National Natural Science Foundation of China (81627901, 81672937, 81773653 and 81803018), Program for HUST Academic Frontier Youth Team (2018QYTD01), Program for Changjiang Scholars and Innovative Research Team in University (IRT13016), Academy of Finland (297580), Sigrid Jusélius Foundation (28001830K1 and 4704580), HiLIFE Research Funds and the European Research Council proof-of-concept grant (decision no. 825020). We thank the Research Core Facilities for Life Science (HUST), the Analytical and Testing Center of Huazhong University of Science and Technology and Wuhan institute of biotechnology for related analysis.

\section{Author contributions}

L.G., H.A.S., and X.Y. designed the project. T.Y., Xiaoqiong Z., N.B., H.Z., Xuting Z., F.L., A.H., and J.H. performed the experiments. T.Y., Xiaoqiong Z., N.B., H.Z., L.G., H.A.S. and X.Y. analyzed and interpreted the data, and wrote the paper 


\section{Additional information}

Supplementary Information accompanies this paper at https://doi.org/10.1038/s41467019-11718-4

Competing interests: The authors declare no competing interests.

Reprints and permission information is available online at http://npg.nature.com/ reprintsandpermissions/

Peer review information: Nature Communications thanks Jeffery Coffer and other anonymous reviewer(s) for their contribution to the peer review of this work. Peer reviewer reports are available.

Publisher's note: Springer Nature remains neutral with regard to jurisdictional claims in published maps and institutional affiliations. (c) Open Access This article is licensed under a Creative Commons Attribution 4.0 International License, which permits use, sharing, adaptation, distribution and reproduction in any medium or format, as long as you give appropriate credit to the original author(s) and the source, provide a link to the Creative Commons license, and indicate if changes were made. The images or other third party material in this article are included in the article's Creative Commons license, unless indicated otherwise in a credit line to the material. If material is not included in the article's Creative Commons license and your intended use is not permitted by statutory regulation or exceeds the permitted use, you will need to obtain permission directly from the copyright holder. To view a copy of this license, visit http://creativecommons.org/ licenses/by/4.0/.

(C) The Author(s) 2019 